\title{
Generative BIM workspace for AEC conceptual design automation: Prototype development
}

\section{Sepehr Abrishami, Jack Goulding, Farzad Rahimian}




\begin{abstract}
:
The integration and automation of the whole design and implementation process has become a pivotal factor in construction projects. Problems of process integration, particularly at the conceptual design stage often manifest through a number of significant areas, from: design representation, cognition and translation, through to process fragmentation and loss of design integrity. Whilst Building Information Modelling (BIM) applications can be used to support design automation, particularly through the modelling, amendment, and management stages, they do not explicitly provide whole design integration. This is a significant challenge. However, advances in generative design now offers significant potential for enhancing the design experience to mitigate this challenge.
\end{abstract}

\title{
Design/methodology/approach
}

The approach outlined in this paper specifically addresses BIM deficiencies at the conceptual design stage. Where, the core drivers and indicators of BIM and generative design are identified and mapped into a G-BIM framework, and subsequently embedded into a Generative BIM (G-BIM) prototype. This actively engages generative design methods into a single dynamic BIM environment to support the early conceptual design process. The developed prototype followed the Centre for Integrated Facility Engineering (CIFE) "horseshoe" methodology of aligning theoretical research with scientific methods to procure Architecture, Construction and Engineering (AEC) - based solutions. This G-BIM prototype was also tested and validated through a focus group workshop engaging five AEC domain experts.

\section{Findings}

The G-BIM prototype presents a valuable set of rubrics to support the conceptual design stage using generative design. It benefits from the advanced features of BIM tools in relation to illustration and collaboration (coupled with BIM's parametric change management features).

\section{Research limitations/implications}

This prototype has been evaluated through multiple projects and scenarios. However, additional test data is needed to further improve system veracity using conventional and non-standard real-life design settings (and contexts). This will be reported in later works.

\section{Originality/value}

Originality and value rests with addressing the shortcomings of previous research on automation during the design process. It also addresses novel computational issues relating to the implementation of generative design systems; where for example, instead of engaging static and formal description of the domain concepts, G-BIM actively enhances the applicability of BIM during the early design stages to generate optimised (and more purposeful) design solutions.

\section{Keywords}

Generative Design, Genetic Algorithm, Space Syntax, AI, Conceptual Design 


\section{Introduction:}

The AEC sector is one of the largest industrial employers, representing $9.8 \%$ of the UK's Gross Domestic Product - employing over $6.6 \%$ of the workforce (Rhodes, 2019). However, industry fragmentation is well recognised, reflected in a number of Government reports. Without wishing to conflate or focus on all issues and forces that have affected this fragmentation, the authors wished to focus on problems relating to design. Where for example, (Elghaish et al., 2020b) highlighted challenges and failures with communication and information processing. These recurrent failures have also (in part) been exacerbated by integration and interoperability challenges held by different stakeholder parties (Pour Rahimian et al., 2019), which supports the intrinsic need to enhance the veracity of design information throughout the project lifecycle from the outset (Goulding and Rahimian, 2012). In essence, the nature and complexity of communication within AEC projects has changed significantly over the last ten years, especially advances in Information and Communication Technology (ICT) and the increased prevalence of: virtual reality-based collaboration technologies (Pour Rahimian et al., 2019); artificial intelligence-based optimisation (Pilechiha et al., 2020); data-driven decision support (Seyedzadeh et al., 2019); smart data modelling (Seyedzadeh et al., 2020); blockchain and distributed ledger technologies (Elghaish et al., 2020a); and computer vision and graphics (Pour Rahimian et al., 2020). Within the AEC sector, ICT has revolutionised production and design (Abrishami et al., 2014a; Pour Rahimian et al., 2008), which has led to considerable changes in labour and skills (Fruchter et al., 2016). Where for example, these advancements are now able to assist decision-making to predict the cost and performance of optimal design proposals (Elghaish and Abrishami, 2020). 
Existing design automation and decision-making support tools/researches mainly focus on form finding/planning and hence, their research is incapable of engaging in information generation during the early stages of design. The research is aimed at leveraging conceptual design automation within AEC projects using BIM integrated generative design, through the development of a proof of concept Generative BIM Workspace for AEC Conceptual Design Automation. The research objectives are; assess and evaluate core drivers and issues regarding BIM, finding an appropriate automation approach for conceptual design optimisation, developing and validating a conceptual framework, developing the proof of concept prototype, and prototype testing and validation. This research makes contributions to the shortcomings of previous research related to automation in the design process (specifically BIM) as well as computational issues related to the implementation of generative design systems that produce meaningful designs. Moreover, practitioners can easily perceive it (reliability) and revise design models (generalisability). Eventually, this framework could supply an extensible structure through which maintenance and revision are made possible. Ostensibly, having a single, flexible and dynamic 3D environment that covers a wide range of architectural design requirements through the whole design process is a vital necessity for designers.

With all above in mind, this paper involves exploring methods in which BIM is employed, not as a representational tool for visualisation per se, but rather, as a comprehensive AEC design support tool for the entire design process. Given this challenge, the specific focus is to improve the conceptual design process by developing a framework that enhances designers' abilities to procure evolving novel and challenging solutions to assist them throughout the process (change management, modification of the model, etc.) a prototype is developed to provide multi proposal designs by using specific inputs such as the correct number of spaces, site boundary, 
and the number of floors for the building with specifying building, the required number of spaces, minimum dimensions for spaces. The main object to produce the design relies on geometry grid which has some characteristics and constraints regarding its cells and faces as well as the interdependences between the grid faces directions and the site boundary. Moreover, there must be accessibility to all spaces from the staircase. Furthermore, the shaped should be simple such as oblong shaped or L-shaped spaces. This process is going to be developed by using Genetic algorithm by using specific routines to transform a set of genes (Genotype) to some specifications which have been affected by the input Genotype (Phenotype). The generative steps will start by defining the Grid positioning with considering the data files that includes the correct number of spaces, site boundary, and the number of floors, and other variables which can affect the spaces characteristics (Janssen, 2006). 


\section{Related Studies}

In AEC practice, designers traditionally tend to explore different design possibilities by producing multiple design solutions, the consequence of which involves additional demand and resources due to independent parallel redundant effort (Succar and Poirier, 2020). One of the key debates in AEC literature on advanced technology adoption is the level of automation required throughout the project lifecycle (Skibniewski, 2014). This challenge also involves integration, which (it is proffered here) tends to be seen as a secondary consideration, and is not therefore embraced until the very late stages of design - where designers start to employ advanced visualisation and modelling technologies.

\subsection{Building Information Modelling}

The implementation of BIM has quickly gathered momentum for its promise of structure, organisation and efficiency of the work processes and tools. The practicality of documenting and exchanging information both as metadata and 3D geometry through unified BIM technologies has now surpassed conventional architectural methods of documentation in the past such as orthogonal drawings and specifications. The benefits of BIM for AEC (including manufacturing) has been widely reported (Abanda et al., 2017; Arayici et al., 2018; Cha and Lee, 2015; Damen et al., 2015). Yet, whilst acknowledging BIM's potential of being able to integrate processes throughout the entire lifecycle of a built asset, there are a number of inherent flaws evident. For example, BIM by its very nature has a lot of semantically rich information embedded into its structural fabric. However, it also has to accommodate diverse data sets from different disciplines throughout consecutive phases; which not only increases file sizes but adds complexity and thus impedes the smooth flow of information exchange. The result of this is that frequent and timely interdisciplinary collaboration is often stifled (or 
discouraged) due to data complexity, cumbersome exchange procedures, large files and mapping requirements involved for achieving interoperability (Arayici et al., 2018). These challenges can sometimes prevent end-users from effectively opening files or navigating them (Walasek and Barszcz, 2017). The corollary of which limits the engagement and decisionmaking processes with clients, owners, stakeholders and other vested professionals (Pour Rahimian et al., 2019).

\subsection{Generative Design}

Amongst the emerging design automation systems, generative ones have been assisting designers to explore design solutions rapidly (Frazer, 2016). That is, they can enhance design by saving time and effort as well as assessing more possible alternatives for the design requirements (Narahara, 2007). Whilst generative tools can assist AEC designers in their design projects, they fail to meet the very basic principles of information modelling and data management requirements (Frazer, 2002). In order to address this problem, for this research, the development of a generative BIM workspace prototype is proposed, which enables design creativity, fluidity and flexibility by the adoption of a generative design approach, whilst exploiting the BIM environment for information generation/management as well as making minimal changes to the common design process. Using such an integrated platform, relevant information to the design requirements can form the system input, whereas the design algorithms can generate the design output. This platform integration can assist designers in solving complex multi-criteria design problems (Narahara and Terzidis, 2006).

The potential of repositories of digital information as drivers for design actions is a key in the research of Ercan and Elias-Ozkan (2015) that explores performance-based design through 
databases, evolutionary algorithms and parametric tools. Their proposed workflow integrates simulation tools and embedded performance measures; thus, it supports the design process where intuition, experience or theoretical knowledge cannot effectively support the decision making.

\section{Research Methodology:}

The research procedure adopted for developing G-BIM prototype is based on the CIFE Horseshoe model (Figure 1). The first stage started with the identification of a problem and finding ways to resolve it (i.e. intuition). After this stage, the points of departure that could address the theoretical gap were detected as well as the key concepts for doing so. This led to the formulation of the research question, theoretical framework, and their corresponding research methods. Following this stage, the research task was classified into the three phases, namely: theory, model, and testing the model. This led to the initial results, which required validation in order to be able to verify the research's potential contribution. The framework development and validation is presented in Abrishami et al. (2014b) and Abrishami et al. (2015). 


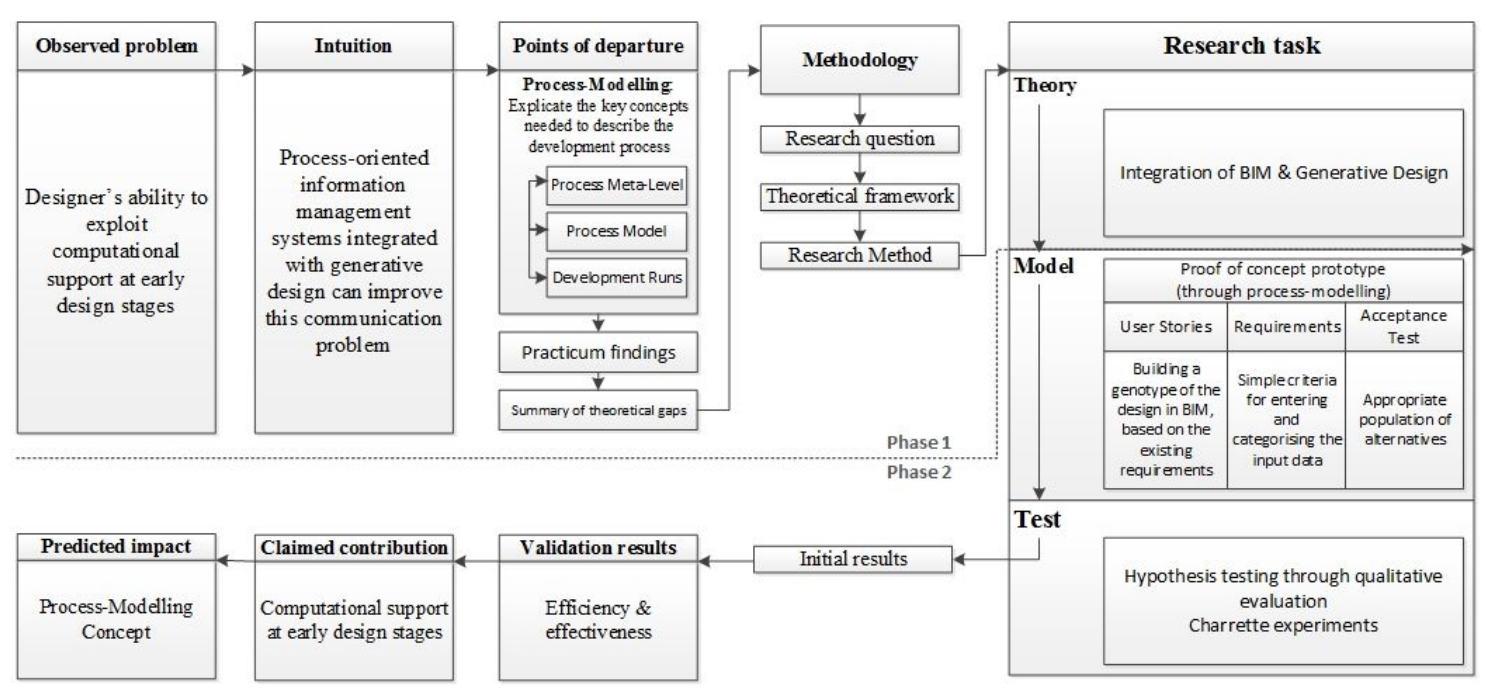

Figure 1: CIFE Horseshoe Research Task: G-BIM

\subsection{Prototype Development Approach}

In this research, the prototype development consisted of two parts: a design method and a computational architecture. The former, pursued at the outset of design process, is aimed at producing a completed building that can tolerate as many future circumstances as possible. The design method broadly defines a design procedure for using generative design to evolve alternative designs. The computational architecture specifies the structure and organisation of the software and hardware components for a generative design system. Such a system is used for the task of evolving alternative designs. This design method involves using a semiformalised design process that explicitly prescribes a way of designing and is structured as a set of tasks to be carried out by the designer or design team, possibly in some specific order.

This research involved following 'Process Prototyping' to develop a multi-disciplinary conceptual prototype. Prototyping has emerged in the world of software engineering as a substitute for the traditional software life-cycle concept (Jacobson et al., 1999). Requirements analysis is one of the early phases in the traditional life-cycle concept. As the term denotes, in 
this phase, the specification of the software requirements is made. Subsequently, software engineers are called upon to generate the ultimate product. In practice, however, it appears completely different as the users' requirements are not sufficiently distinct and constant at the early stages; consequently, the developers' misunderstandings plus enduring changes over time can lead to the emergence of mistakes. As a result, with the Software Development Process Prototyping concept, it is assumed that from the beginning there are a consistent number of requirements that will not change during the development process.

One of the major problems in relation to the analysis and requirements of systems, is the lack of a clear idea about the end-user's expectation from them, so very often the needs are not adequately communicated (Jacobson et al., 1999). Moreover, the end-users seek satisfaction of functionality, ease of application, and compatibility to their needs, which might be difficult to gather in the form of a written specification. Thus, forward thinking is required to be able to incorporate user specification in the development process. Having released the final prototype into the phase of testing and validation, the developer will normally receive a myriad of requests for alterations, many of which require performing the software design and analysis process all over again. As a result of this process, and due to these late changes, further interruption in the accessibility of the final prototype and potential loss of system structure can occur. consequently, through the development process, methods permitting quick construction and modification of system prototypes would be the preferred options (Wasserman et al., 1986). For example, the users are provided with a prototype of the user-program interface. Thus, they are able to evaluate it to find out about the availability of all the desired functions and whether the dialogue has an accessible format. By using a dialogue processor as well as a system of database management, a useful prototyping tool can be generated for programs, 
which enables conversational access to databases. Overall prototyping involves the following steps, namely: planning, development, testing, and documenting, which are explained below:

- Planning: Distinguishing basic requirements, such as the desired input and output information. Some details, like security, are usually ignorable (phase one in Figure 2);

- Development: Generating the primary prototype, which comprises only user interfaces (phase two in Figure 2);

- $\quad$ Testing: Reviewing the prototype and providing feedback on additions or changes (phase three in Figure 2);

- Documenting: Improving the prototype by making use of the feedback; it is essential to discuss the contents of the product (phase four in Figure 2).

\subsection{Prototype validation Approach}

Focus group has been applied as the key research activity in prototype validation (Parker and Tritter, 2006). Focus groups are substantially different from surveys and questionnaires, mainly on account of the fact that not only do they enable the collection of data on G-BIM prototype use, but also, they provide a forum for the contrasting disciplines within the AEC industry. This will allow them to share and elucidate their views on different G-BIM adoption issues, the main examples of which would be common understanding of benefits, hurdles, requirements and expectations (Morgan, 1997). For conducting the focus group, five experts in the UK from the field of AEC design were recruited. The focus group lasted two sessions. During the first, the experts were given a design brief and asked to design a project based on the conventional design methods. In the second session, they were introduced to the G-BIM prototype and asked to classify the design brief as well as to import what they considered to be the information into the prototype. Later, they selected the preferred design alternative amongst the final population, after which they were able to modify the selected design according to their own preferences. Finally, the participants were asked to compare the conventional method of design with the design aided G-BIM prototype and state their opinions about the latter. 


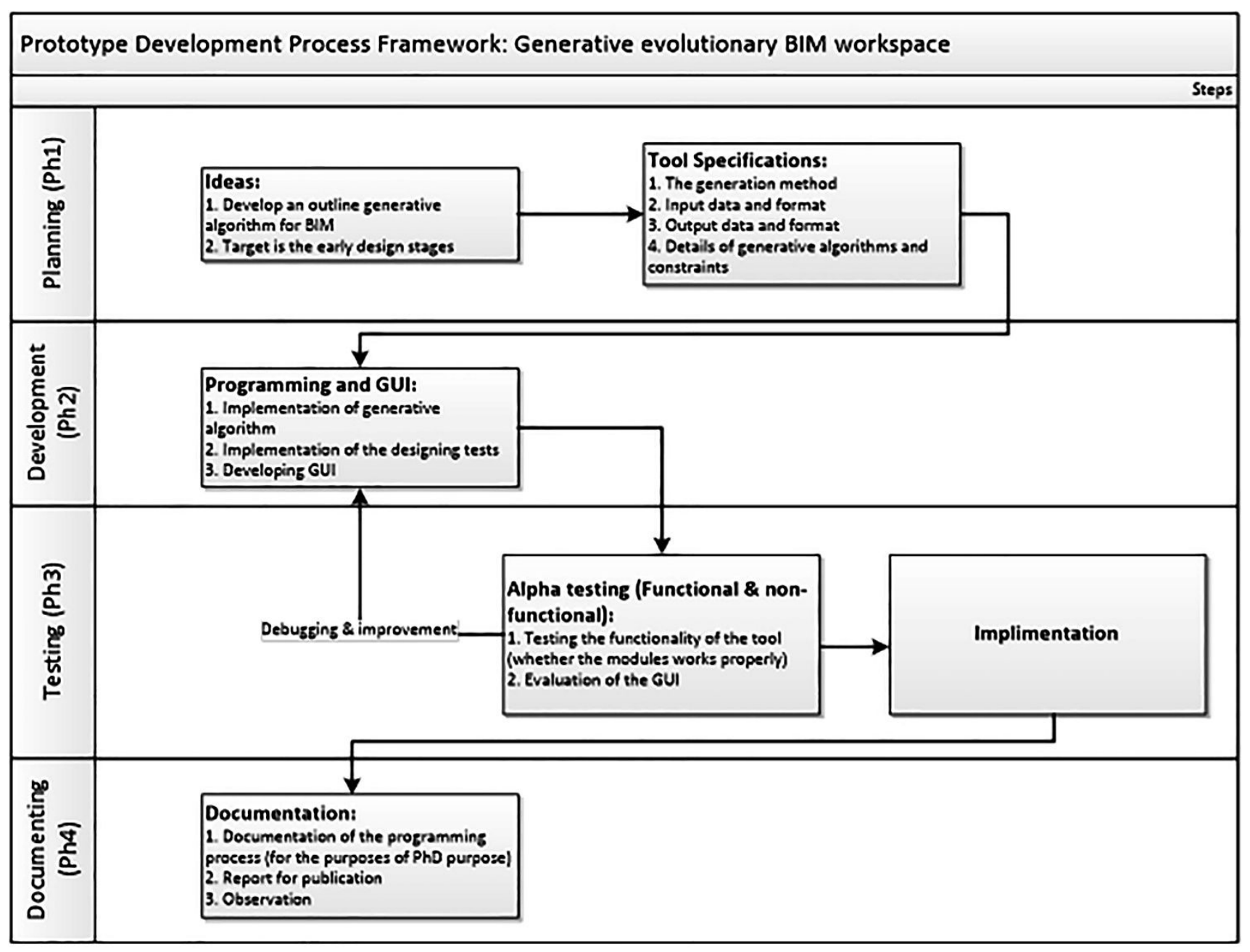

Figure 2: Prototype Development

\section{G-BIM Prototype Development}

The overall generative process is centred on the gradual modification of an orthogonal threedimensional grid, consisting of a set of cuboid cells, the dimensions of which are predefined as part of the schema and are set at 3.0 metres. The environment data-file contains the specification of the length, width, and height of the grid in the cells. In order for the generative process to be clarified, a number of terms associated with the grid, need to be defined first. The grid consists of three main types of entities, namely, grid-faces, cells, and spaces. Cells and spaces may be further broken down into cellfaces and space-faces, respectively. Grid-faces are referred to as a set of intersecting quadrilateral 
surfaces that define the grid and they are naturally perpendicular to the Euclidean $\mathrm{x}, \mathrm{y}$, and $\mathrm{z}$ axes. Cells are defined as spatial units within the grid, each of which has six cell-faces, which are parallel to the grid-faces.

Spaces are defined as larger spatial units that consist of one or more cells. Providing that the space is oblong shaped, it will equally be defined by six space-faces. Nonetheless, it is highly probable that spaces also have more complex shapes, which will end in there being more space-faces. More importantly, the space-faces are coplanar with the cell-faces, and each space-face will encompass one or more cell-faces. Every single grid- face should have a front that faces in the positive direction of the axis perpendicularly, and a back facing in the negative direction. There are grid-faces that are in line with the ground and are referred to as horizontal grid-faces, having a top grid-face being the roof gridface, and a bottom one called the floor grid-face. Grid-faces that are not in a horizontal manner are referred to as vertical grid-faces. There also is a distinction between inner grid-faces that possess adjacent grid-faces on either side and outer grid-faces that are characterised by having only one neighbouring grid-face. Based on Janssen (2006) proposed approach, there are seven sequential generative phases a generative process consists of: positioning of the grid, the grid-faces translation, the outer grid-faces inclination, the staircase insertion, spaces creation, insertion of the doors and windows. A set of parameters is required by most of the phases to be encoded within the genotype, each always being encoded as real values in the range 0.0 to 1.0. Furthermore, it is likely that the encoded value be mapped to a value within a varied continuous range by the generative step, as required. Some steps may require extra parameters or data being encoded in the environment data-file.

\subsection{G-BIM development process prototyping}

The development step is aimed at providing a tool for automation during the conceptual design stage using a genetic algorithm. This phase of the research was divided into two parts:

- Stage 1: Creating a suitable design environment capable of satisfying primitive 
conceptual design requirements, i.e. walls, doors, windows etc. and with the capacity to provide information embedded within the design by executing genetic programming;

- Stage 2: Developing a method for conceptual design automation, based on the integration of BIM and a genetic algorithm.

One of the most advanced BIM applications is Autodesk Revit and one of the capabilities of the Revit environment is exerting control over 3D modelling commands. This control is based on user inputs using the C\#.Net programming language. Autodesk designed the Revit API in the form of Addin for connecting the tools provided by developers to the Revit environment. The API is based on the C\#.Net programming language and in the context of this research, the Revit architecture has been used for GBIM development. The development requires knowledge of C\#.Net programming in the domain of Autodesk Revit Addin plus familiarity with the C\#.Net functions. These functions have been developed by Autodesk and presented through Revit API to the developers. Moreover, the developers are required to possess considerable knowledge of the Revit functions and their capabilities for architectural design. The software prototyping method is applied in the development phase of G-BIM. Using this method, the process of developing is divided into several stages and at each stage a basic prototype based on the G-BIM framework is constructed. These prototypes only represent a few functions of the final one. At each level of the development process, the prototypes are analysed and tested to ascertain whether they fulfil the requirements for the next stage of the software development process prototyping. Following sub-sections describe the seven stages of the prototype development.

\section{Stage 1: Initial prototype during the development phase}

The initial prototype during the development phase of G-BIM was formed as follows. A basic Addin with the capability of generating a simple building up to three levels was developed, which was used to examine the feasibility of the conceptual design automation within the Revit software using Autodesk 
Addin capabilities. The user could input the number of levels (up to three), width, and length as well as the number of doors and windows. Running the develop Addins within the Revit architecture was naturally done by putting the .Addin file in the designated Autodesk Addin folder and addressing the main .dll file in the .Addin folder. This file was developed using C\#.Net and this process is the main G-BIM algorithm. Figure 3 illustrates a sample of a .dll file.

Figure 3 identifies the SimpleHouse.dll, which was the initial prototype integrated within the Revit environment. After running Revit, the user would need to choose the simple house from the Addin menu. When running the SimpleHouse, the user interface opens and requires some parameters to be manually inputted (house length and width, number of levels, level heights). By confirming these parameters, a simple house based on the user input is generated. Figure 4 presents a screenshot of this developing stage. The following stages list the initial prototype development process:

1. The initial prototype confirms the feasibility of the development and integration of G-BIM within the Revit environment;

2. The possibility of developing a user-friendly interface with the capability of accepting user inputs and integrating them within the Revit environment along with the design generation has been tested and analysed;

3. The DialogForm has been used for initial prototype development;

4. The next phase of the prototype development is to be designed based upon the results obtained from the initial phase. 


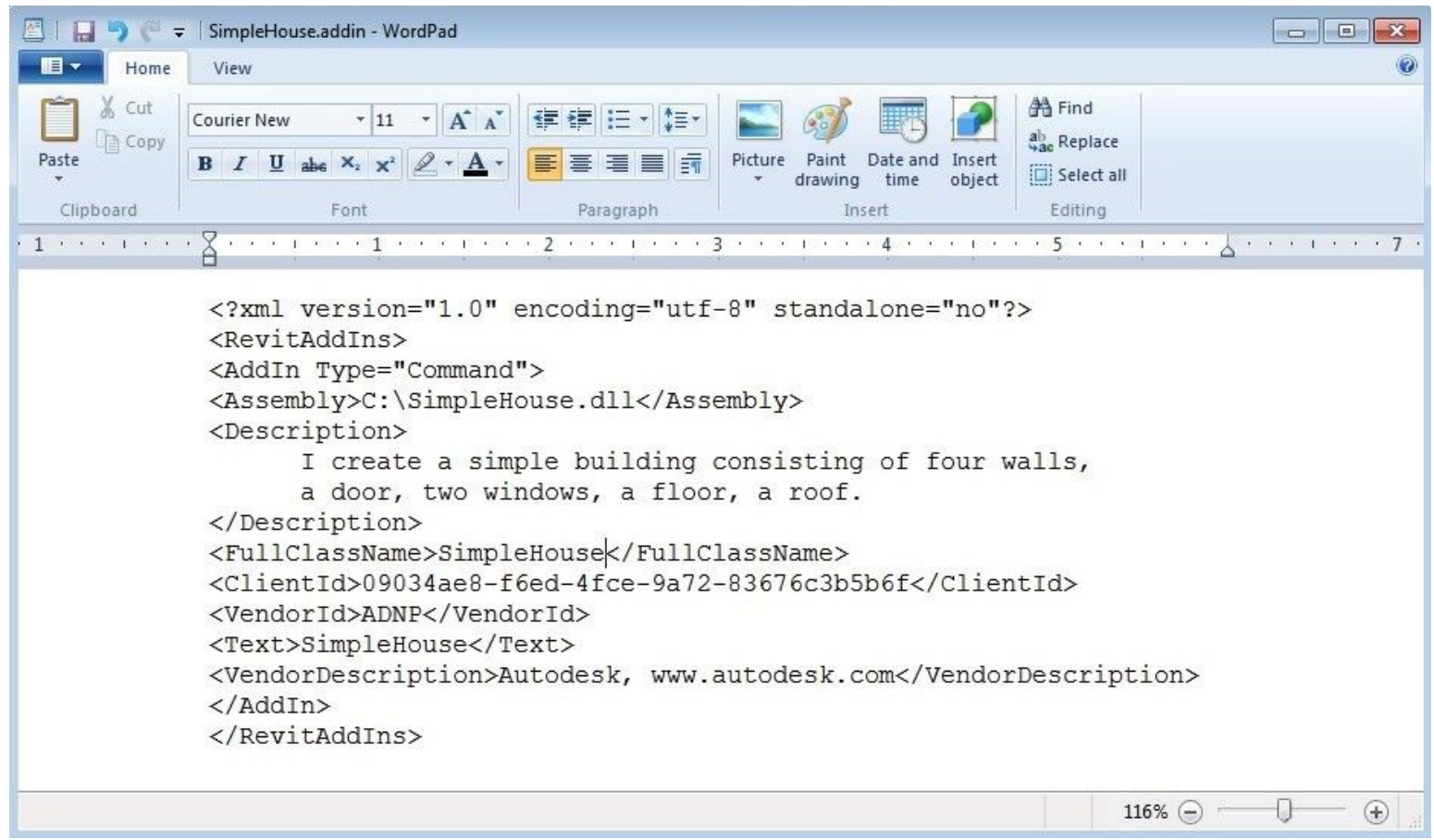

Figure 3: A sample .Addin File

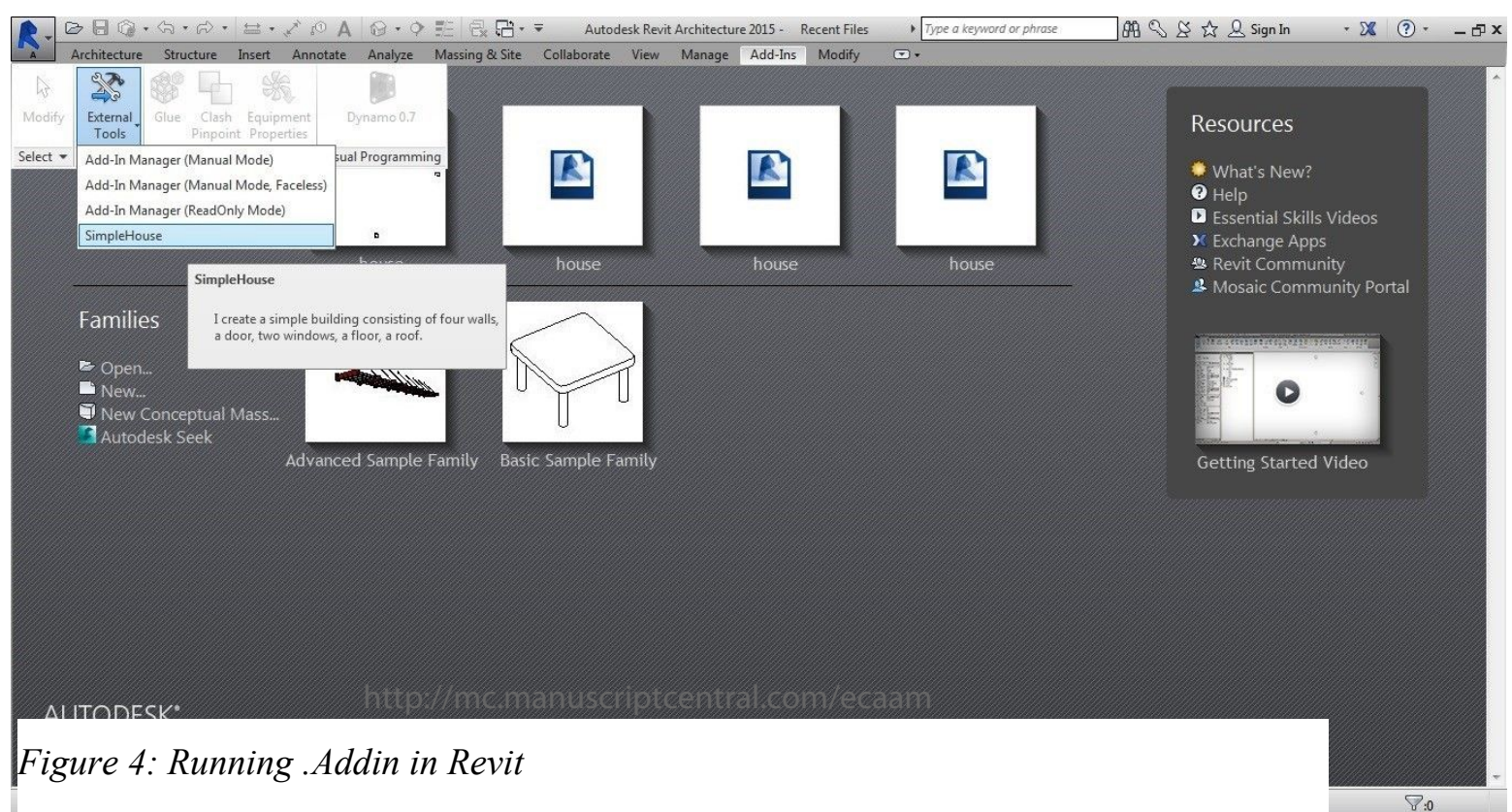




\section{Stage 2: Process prototyping phase}

The second phase in the process of software prototyping focused on the possibility of form generation based on grids, orientation and relocation of the form defined by the site inputs, as presented in the $\mathrm{C}$

5. The input form was expanded to accept vertical and horizontal grid numbers, rotation and location of the design. The following statements arose from the second cycle of the prototype development process:

1. Some cases of individual face rotation have caused detachment of the form;

2. The possibility of generating sloped walls (orientation towards $\mathrm{x}$ axis) is not considered in Revit API platform;

3. The DialogForm has been used at this stage;

4. Based on the results of this stage the next step of the software prototyping process is formed.

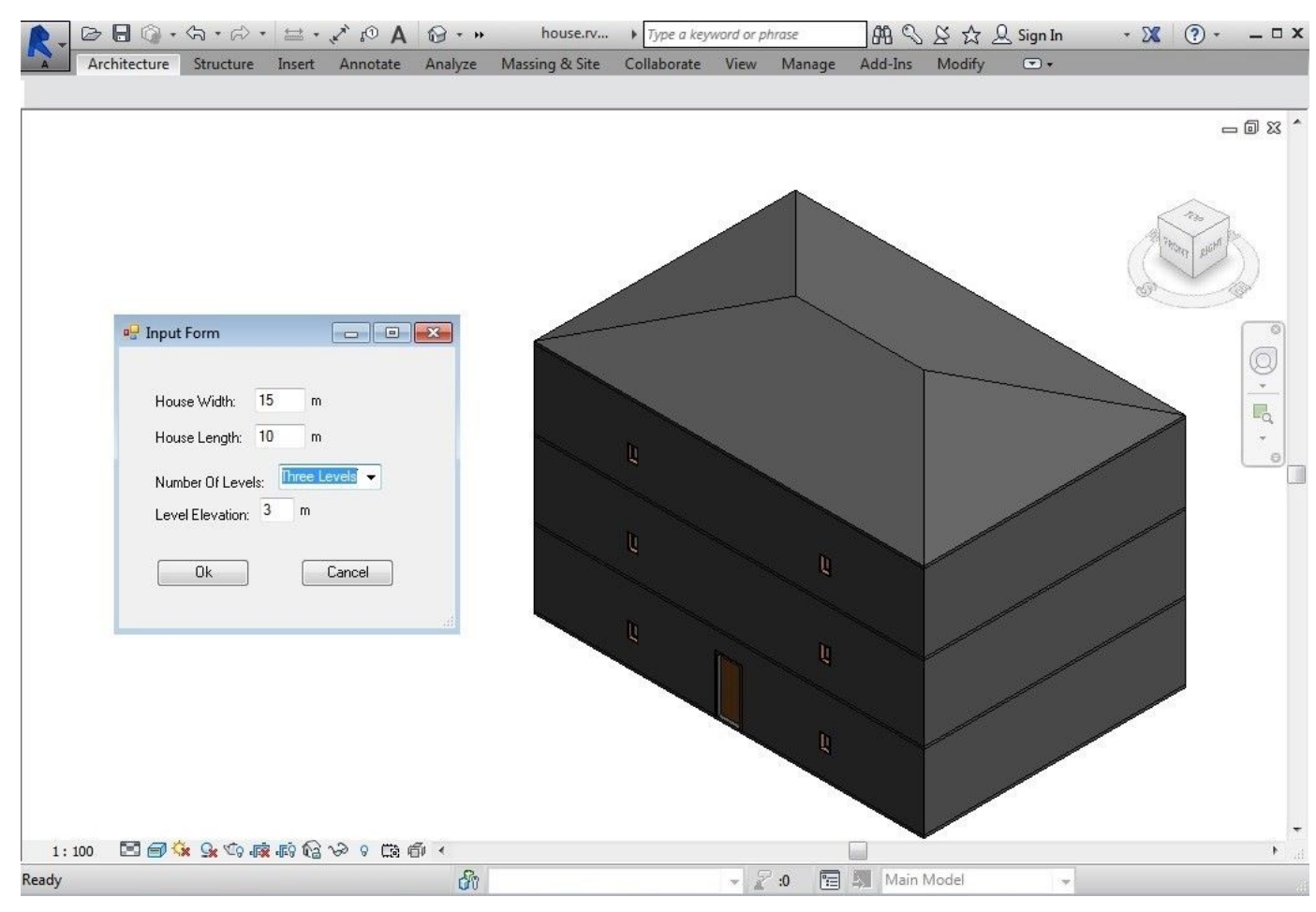

Figure 5: Initial Prototype User Interface 


\section{Stage 3: Staircase generation}

The third phase of the development was primarily aimed at generating the staircase based on the user input data. Determining the width, length, height and accessibility of the staircase at each level of the building was an important aspect, which needed to be considered in staircase generation using the Revit API. The user inputs at this stage consisted of vertical and horizontal face numbers as well as the width, length, heights and number of levels. Figure 6 is a screenshot of this stage of the development. The following statements are made pertaining to the third phase of the software prototyping process:

1. The staircase has been generated automatically within the building borders and in this prototype (Figure 7);

2. As shown in Figure 8, an opening connected to the stair box has been created at the top level to make the roof of the building accessible;

3. Based on the level heights, the prototype has generated the staircase, which is accessible at each level of the building;

4. The DialogForm has been used at this stage;

5. Based on the results of this stage the next step of the software prototyping process is formed.

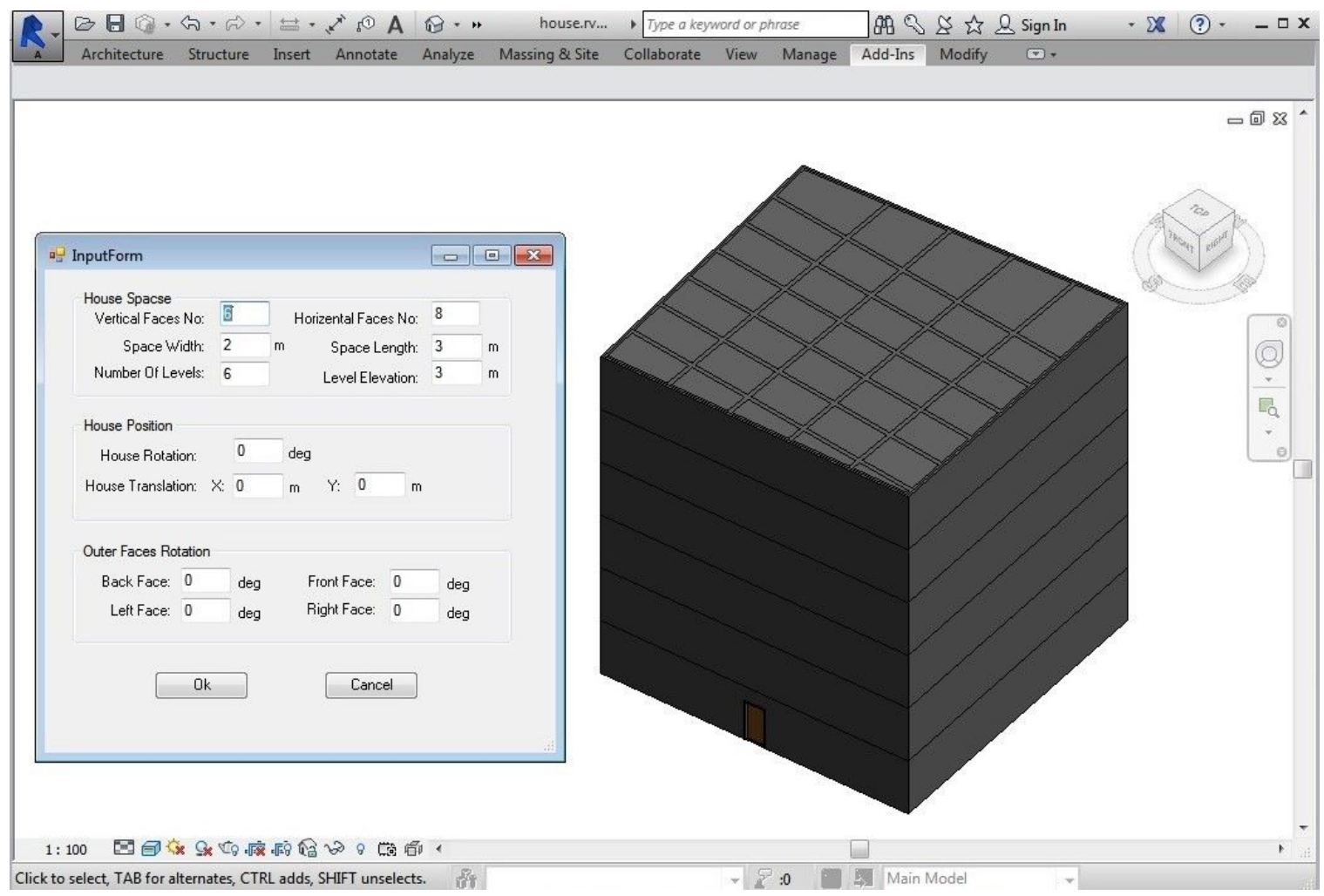


Figure 6: Input-form and a generated design from the third phase of prototype development

\section{Stage 4: Room generation}

The central aim of the next phase of development was generating rooms and specifying the location of the doors and windows using the Revit API. As can be seen in Figure 9, the user inputs were: vertical and horizontal face numbers, widths and heights of each level, the number of levels, the building rotation around the $\mathrm{z}$ axis and the building location. Figure 9 depicts the building generated within this phase of the prototype development. The following statements are made regarding the third phase of the software prototyping process:

1. Doors and windows are assigned to every space created within this prototype;

2. The DialogForm has been used at this stage;

3. Based on the results of this stage the next step of the software prototyping process is formed.

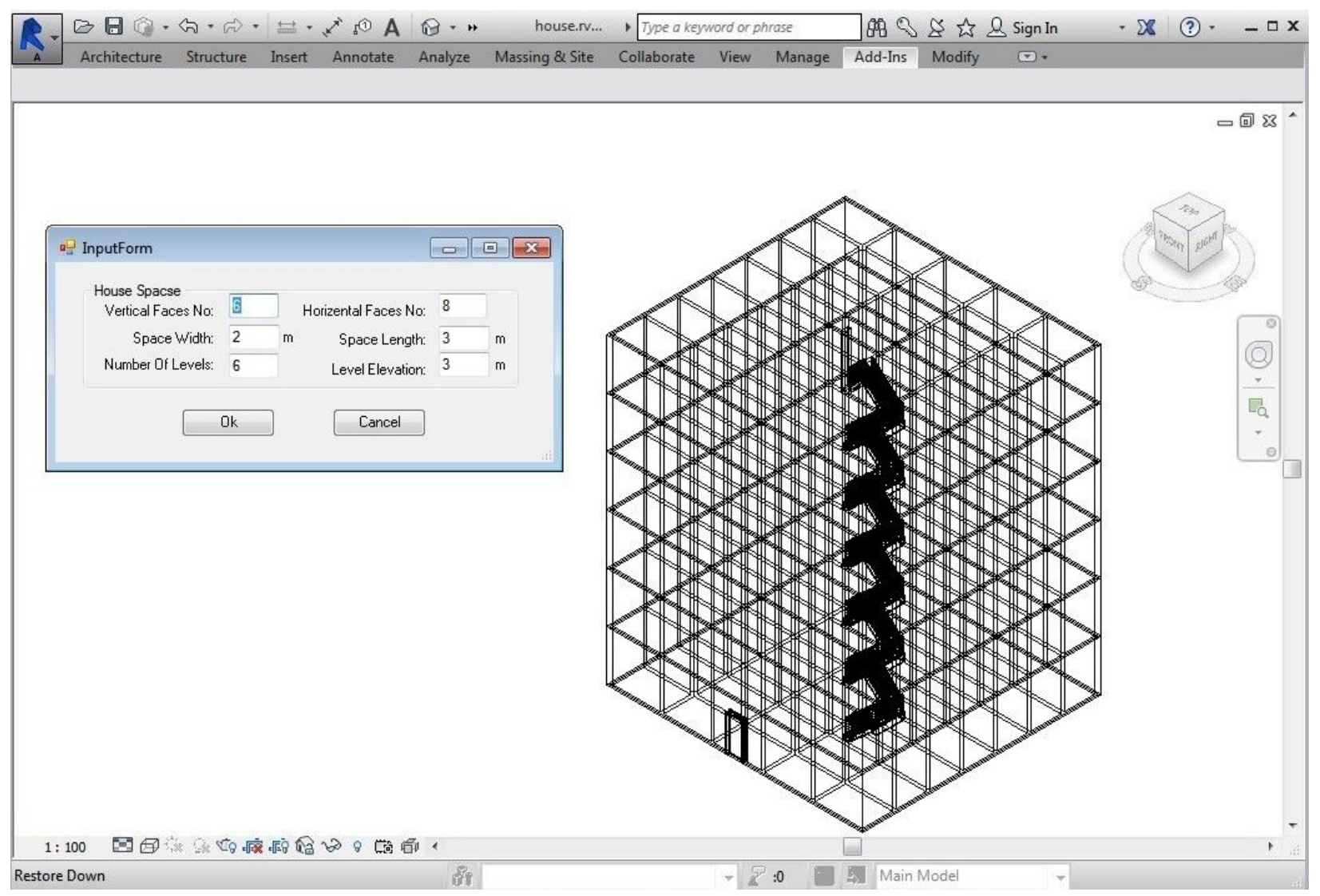


Figure 7: Input-form and a result from the third phase of prototype development (in wireframe mode) 


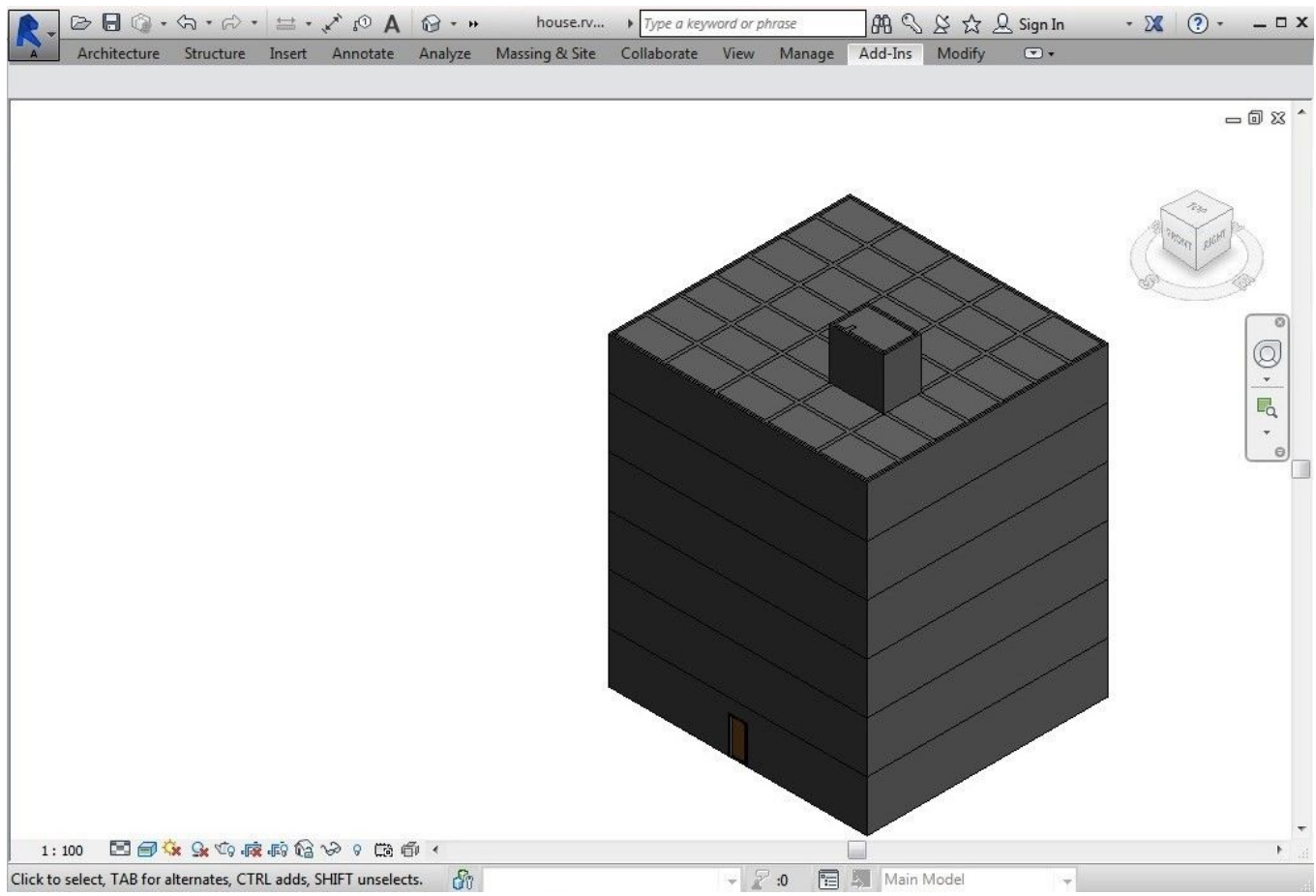

Figure 8: Positioning of the Stairbox in 3D View

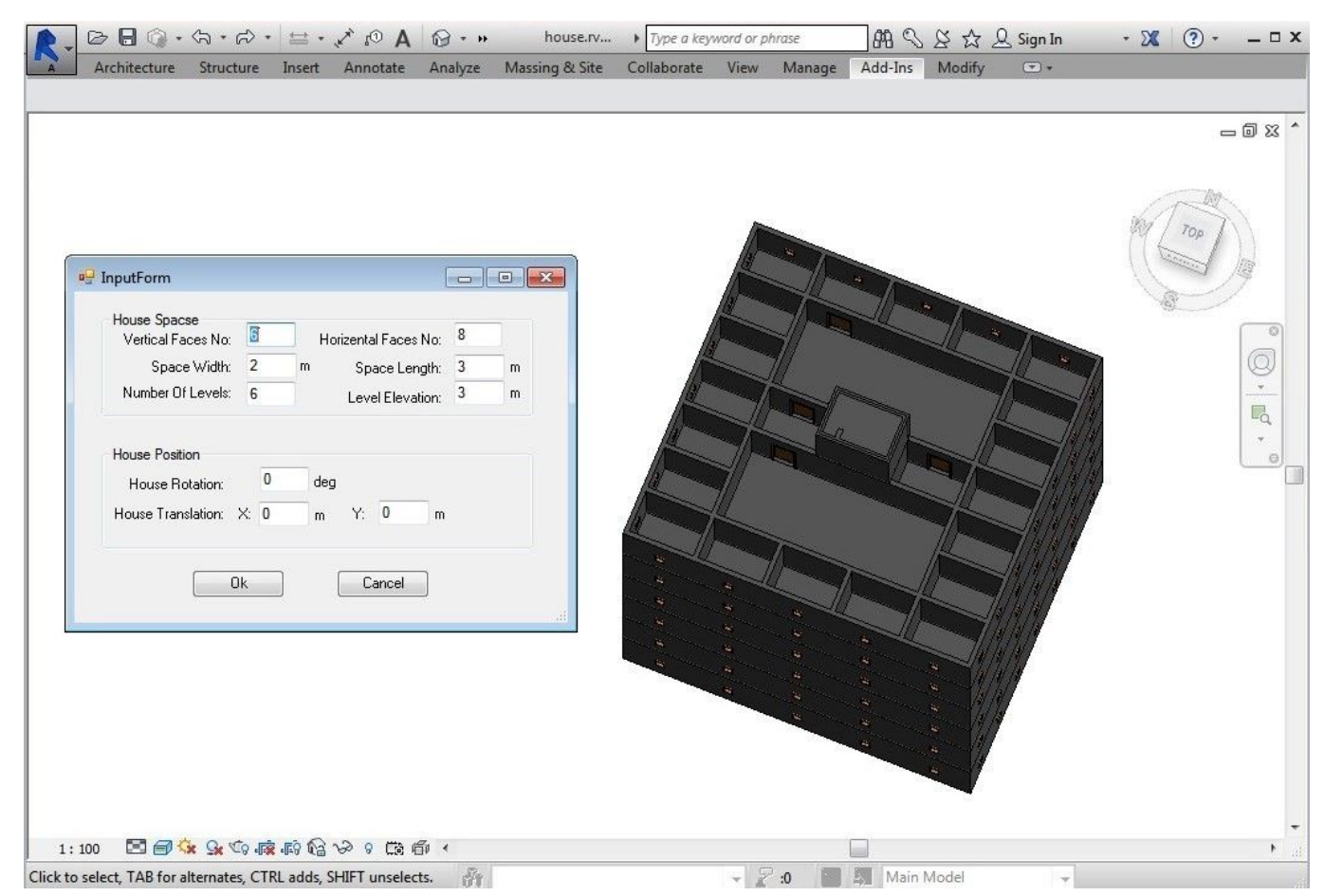

Figure 9: Input-form and a result from the fourth phase of prototype development 


\section{Stage 5: Generation phase}

After testing and validating the method for creating the primitive elements of the building (such as the walls, windows, doors and staircase) using Revit API, the next stage of the prototype development was focused on an intelligent method based on a genetic algorithm for the generation of the building. As is evident from Figure 10, in order to analyse the possibility of integration of BIM and the genetic algorithm, the user inputs within this phase of development were significantly simplified. During this stage, the width and length of the spaces and location of the staircase were assumed to be fixed. Moreover, the selection method and mutation probability within the genetic algorithm was fixed. In the genetic algorithm, the overall building area and number of spaces determined the initial population size. Consequently, expanding the area and/or number of spaces increased this size. In addition, the connections between the spaces and number of windows were two contributing factors in the evaluation of the fitness function. The criteria for the genetic algorithm are:

1. Calculation of the minimum area required based on the number of spaces (user input);

2. Dividing the building area into equal sized grids;

3. Random generation of spaces based on the grids generated in the previous level and consequently, each 2D matrix forms a chromosome;

4. Repeating level three until the initial population size reaches the number specified by the user;

5. The evaluation of each chromosome's fitness function from the initial population is undertaken by considering the following criteria:

(a) As a result of random generation of spaces, these are randomly scattered all over the place. Therefore, decreasing the standard deviation of the related spaces, groups the related spaces in neighbour grids. In other words, a lower standard deviation results in higher fitness. The standard deviation is calculated for each individually generated space and their summation is negatively related to the fitness value of each chromosome;

(b) In calculating the chromosome value, the generated design alternatives with more connected spaces (neighbour grids) of the same category possess a higher fitness number. The categories are specified based on the user input;

(c) The number of openings plays an important role in the evaluation of the chromosome 
6. After the calculation of each chromosome value, those with higher value are chosen using tournament selection;

7. Given the possibility of scattered spaces all over the building and the algorithm structure, cross-over cannot be performed on any two chromosomes, but it is feasible to alter any chromosome's matrix. This alteration is performed by randomly swapping some rows and columns within the matrix;

8. In order to apply mutation, in each cycle, with fixed mutation probability, two numbers (submatrix) are selected and exchanged randomly at a constant rate;

9. Returning to level five and going through all the levels until the desirable outcome is achieved.

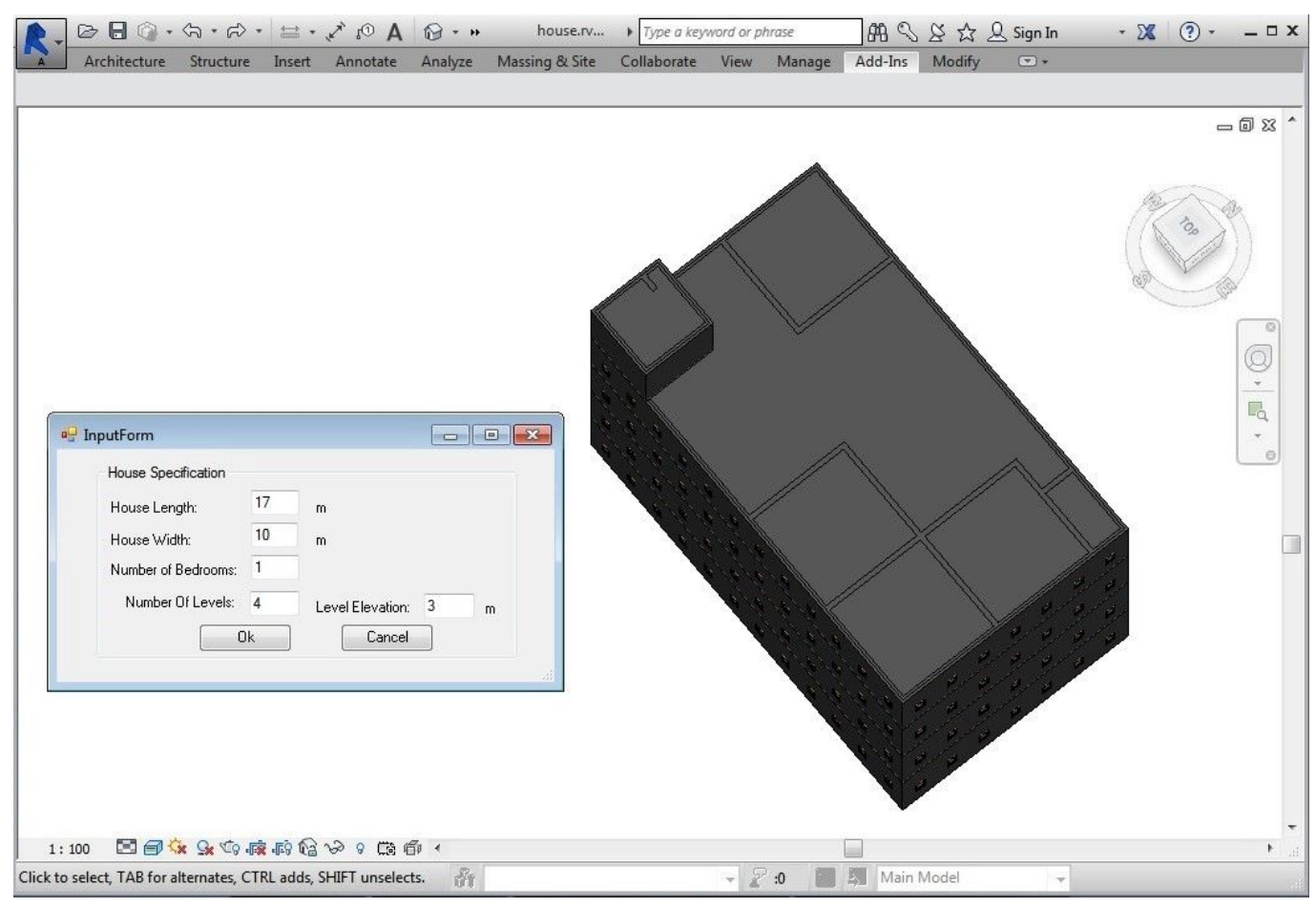

Figure 10: Input-form from the fifth phase of prototype development 
The following statements are given based on this stage of prototype development:

1. The possibility of integrating artificial intelligence (generative design) with BIM has been investigated and validated. This prototype has led to the arrival at an optimal solution to the design problem;

2. From Figure 11, the generated building consists of four rooms, a bathroom and a toilet. As can be seen, one of the rooms is trapped between the other two rooms, not being connected to the corridor, which reveals one of the deficiencies of this algorithm;

3. Problem-solving speed plays an important role in the evaluation of algorithm efficiency. By increasing the building size and/or number of spaces, this method increases the initial population and the chromosome's matrix size. Consequently, more time is required to reach the optimal solution and hence, one of the main problems associated with this algorithm is its low speed;

4. Another deficiency of the developed algorithm is its inability to produce significantly different designs (chromosomes) in the final population. This occurs due to it being trapped in a 'local minima', which results in it focusing on a particular optimisation, thereby losing its ability to generate chromosomes that are significantly different from each other;

5. DialogForm was used in this stage of prototype development;

6. Based on the results obtained in this stage, the next step of the software prototyping process is formed according to the following statements:

(a) During the next prototype development stage, GA parameters, such as the mutation probability will be altered to avoid the algorithm being trapped in a 'local minima';

(b) The user will be able to enter more information input, such as the sizeof spaces, initial population, etc.;

(c) The user will be able to enter new parameters manually;

(d) The user will be able to specify the final population number;

(e) The 'Progress Bar' is to be added to the user interface. 

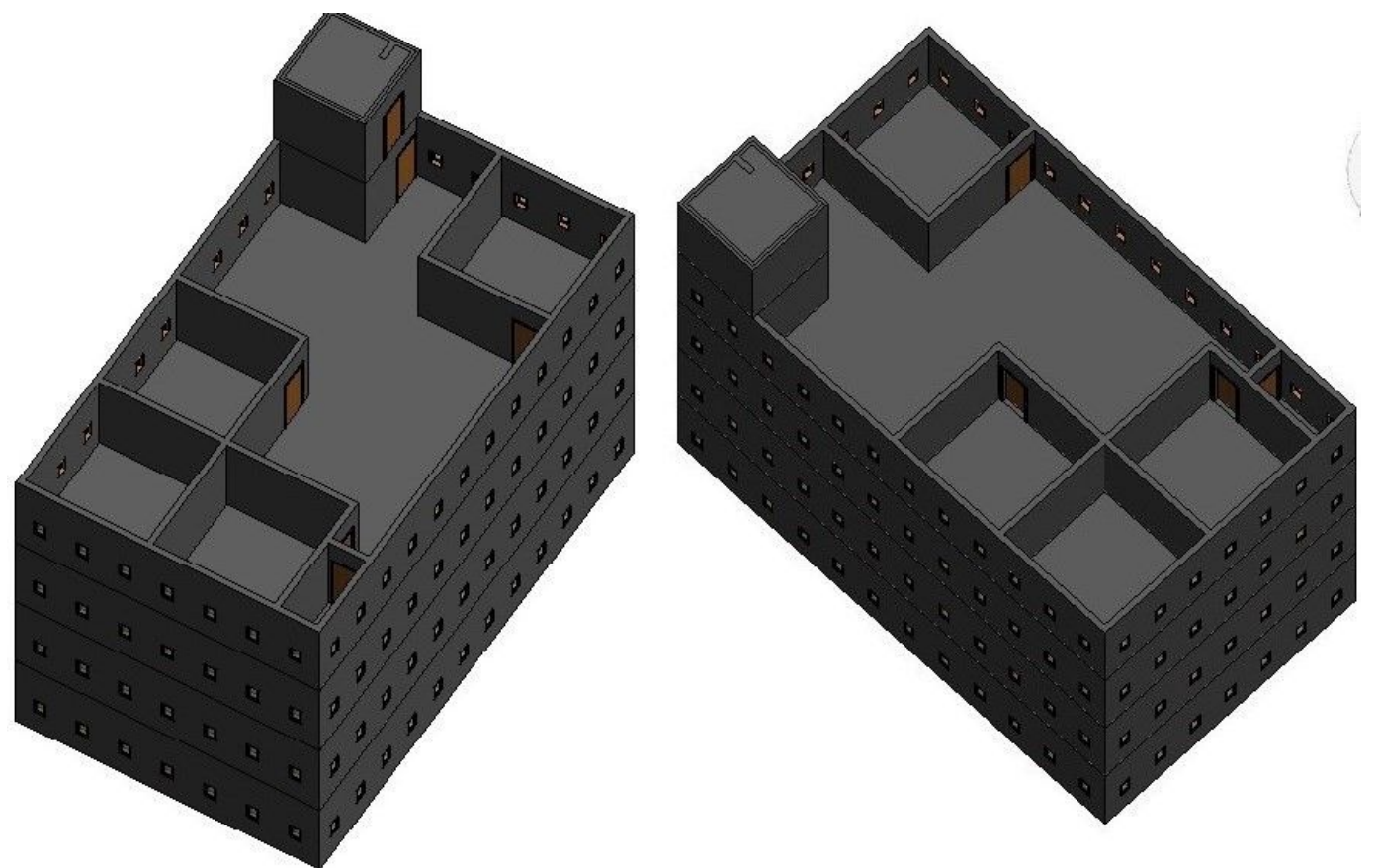

Figure 11: A generated design based on the genetic algorithm from two different perspectives

\section{Stage 6: Design input}

For the sixth phase in the software prototyping process the user input informationincludes: length, width and the number of different spaces as well as their area and level of elevation. Furthermore, some predefined design specifications are added to the prototype, including: the kitchen is to be centred; the restroom(s) are to be remote from the kitchen, whilst the restroom(s) and stair cases are to be next to each other; the private, semi-private and non-private areas are to be separated from each other; and the staircase is to be central. As mentioned before, the initial and final population size is to be entered by the user. The following statements are given based on this stage of the prototype development:

1. The 'local minima' is avoided in this phase of prototype development, which has been achieved by altering the mutation rate. Figure 12 shows a screenshot of the final population, with the roofs being hidden in order to provide a better understanding of the generated alternatives;

2. The user can enter the size of each area;

3. "House Design Parameters" are added as optional constraints; 
4. The user can keep track of the generation progress using the progress bar;

5. The algorithm speed in solving problems is still one of the primary problems within this stage;

6. DialogForm has been used within this stage of the prototype development;

7. Based on the results of this stage the next step of the software prototyping process is formed based on the following statements:

(a) DialogForm is to be omitted in the next phase of the prototype development and consequently, the user will be able to re-run/re-use the algorithm as well as changing the parameters;

(b) A new algorithm will be developed in order to solve the algorithm speed issue;

(c) The final population will sorted by an alternative fitness value.

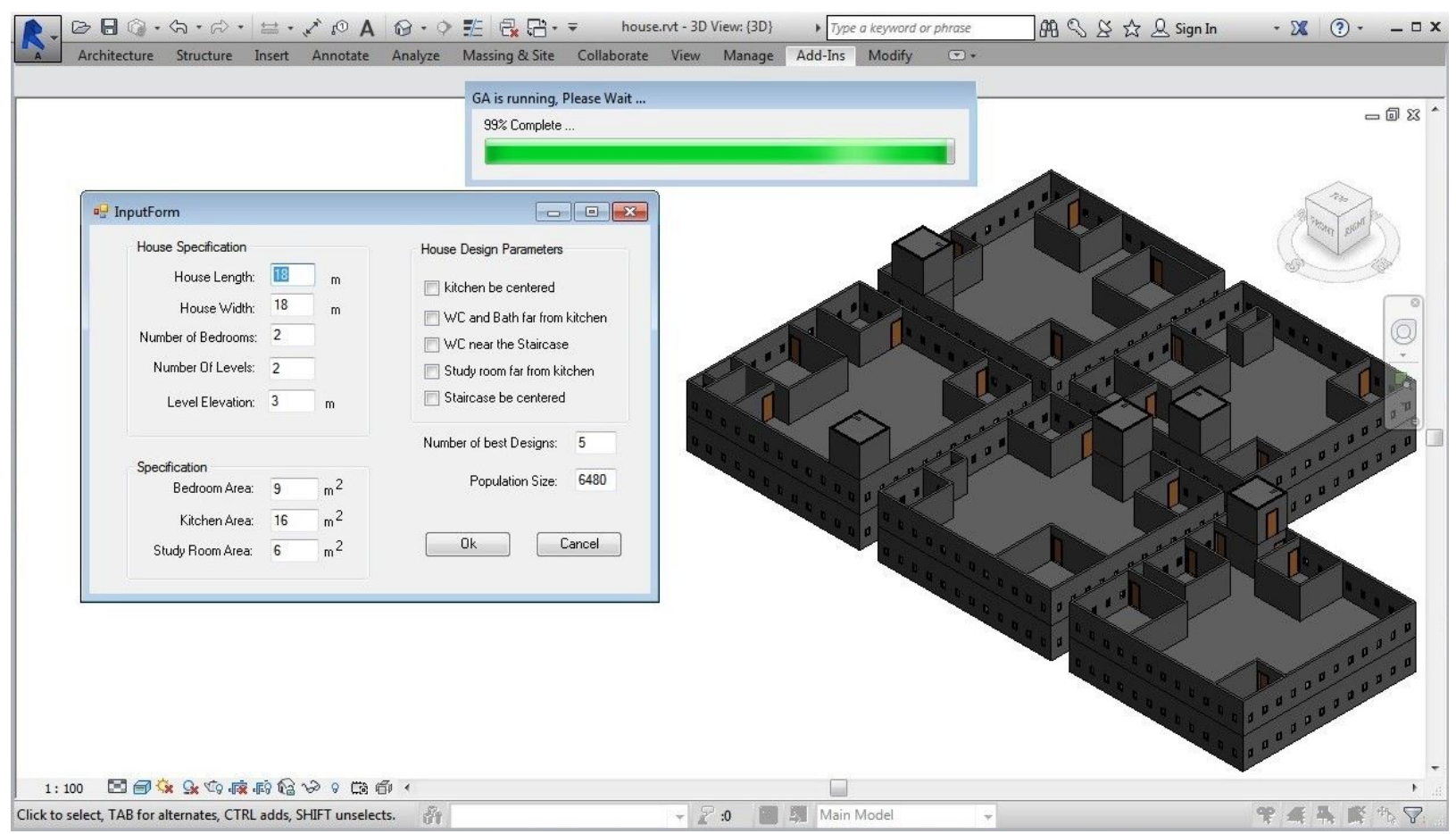

Figure 12: Input-form and results from the sixth phase of prototype development 


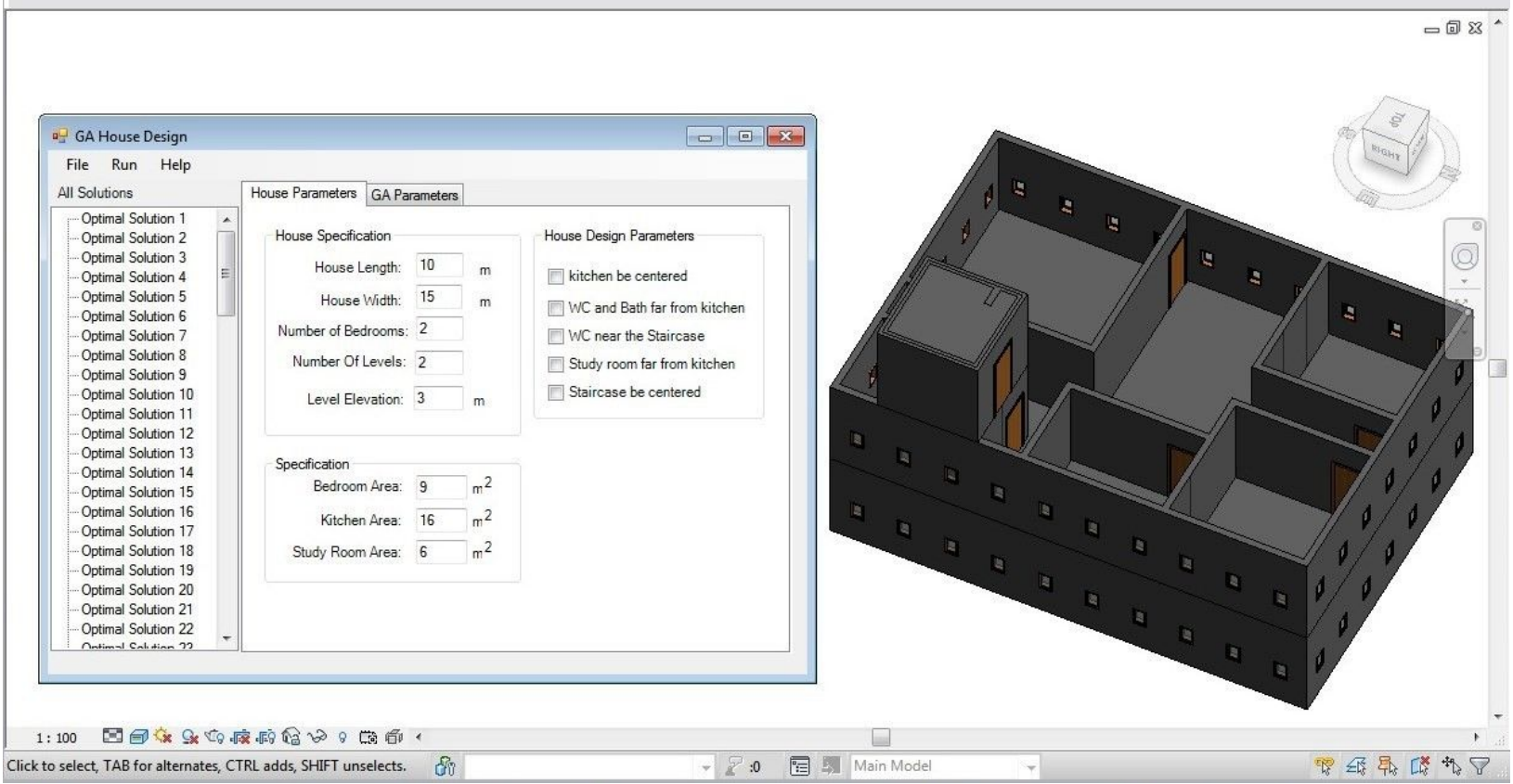

Figure 13: Input-form from the seventh phase of prototype development (with the House Parameters feature)

\section{Stage 7: Space planning}

Regarding the seventh phase in the process of software prototyping the user-interface is redesigned, as can be seen in Figure 13. A taskbar similar to the Revit taskbar has been added with more capabilities. Moreover, the option to save any criteria in any stage of the generation is added to G-BIM, which can be exported/imported or run later on by the design team. Also, a help option has been added in the menu

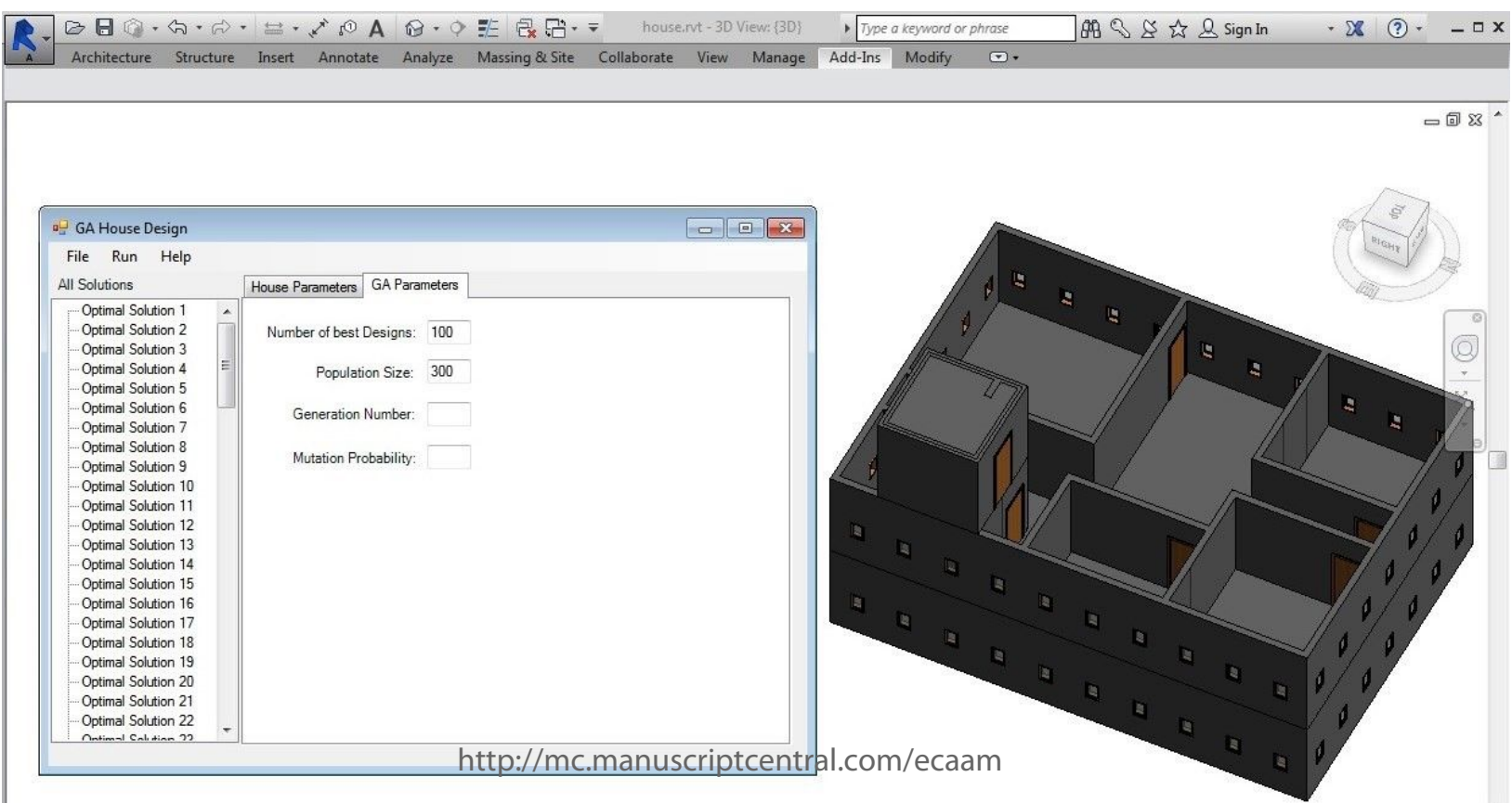

Figube 4: Input formafrom the seventh phase of prototype development (with the GA Parameters feature) 
for guidance purposes. As can be seen in Figure 13, a 'TreeView' is added which sorts the final population based on their fitness value and the user can view any alternative from the TreeView list by clicking on it. Two 'TabPages' are available on the G-BIM user interface that enable the user to modify GA and the design parameters (Figure 14). The following statements are given based on this stage of prototype development:

1. A 'Space Planning' method, as described in subsection 5.3.2, is adopted in order to increase the algorithm speed and accuracy. The algorithm functions as follows:

(a) Calculating the minimum space requirement based on the user input. If the minimum space meets the site data, the algorithm proceeds to the next step, otherwise it stops;

(b) Defining the grids (subsection 5.3.3) based on the site-data. Grid-faces are referred to as a set of intersecting quadrilateral surfaces that define the grid, which are naturally perpendicular to the Euclidean $\mathrm{x}, \mathrm{y}$, and $\mathrm{z}$ axes;

(c) Allocating a matrix to the building with a certain number of columns and rows interrelated with the number of spaces;

(d) Randomly assigning the matrix rows to building spaces' centre and this 2D matrix acts as a chromosome in GA. A certain number of 2D matrixes interrelated with number of spaces will be produced equal to the initial population number (user input);

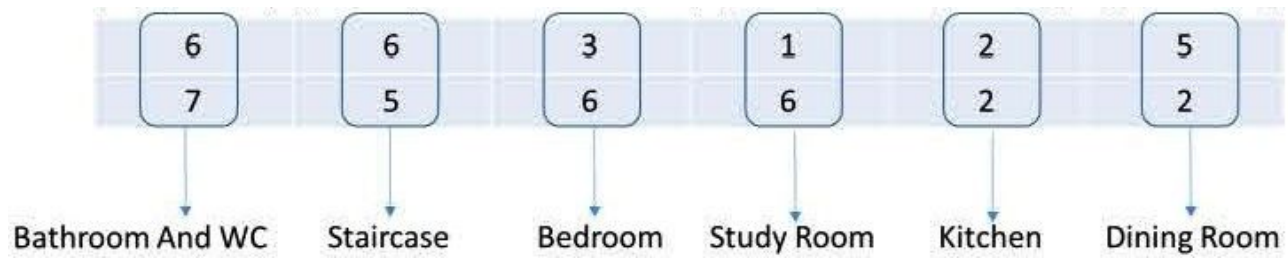

(e) Evaluating the initial chromosome population using the 'Heuristic Evaluation Function' and generating a 'vector-valued function' for each chromosome:

i. Each single space (required by the designer) is assigned to a submatrix formed around the specific space centre. These submatrices act in the same way as the gridding system. Some matrix elements might overlap and be assigned to more than one space due to the regeneration process of a matrix that has already been assigned to spaces;

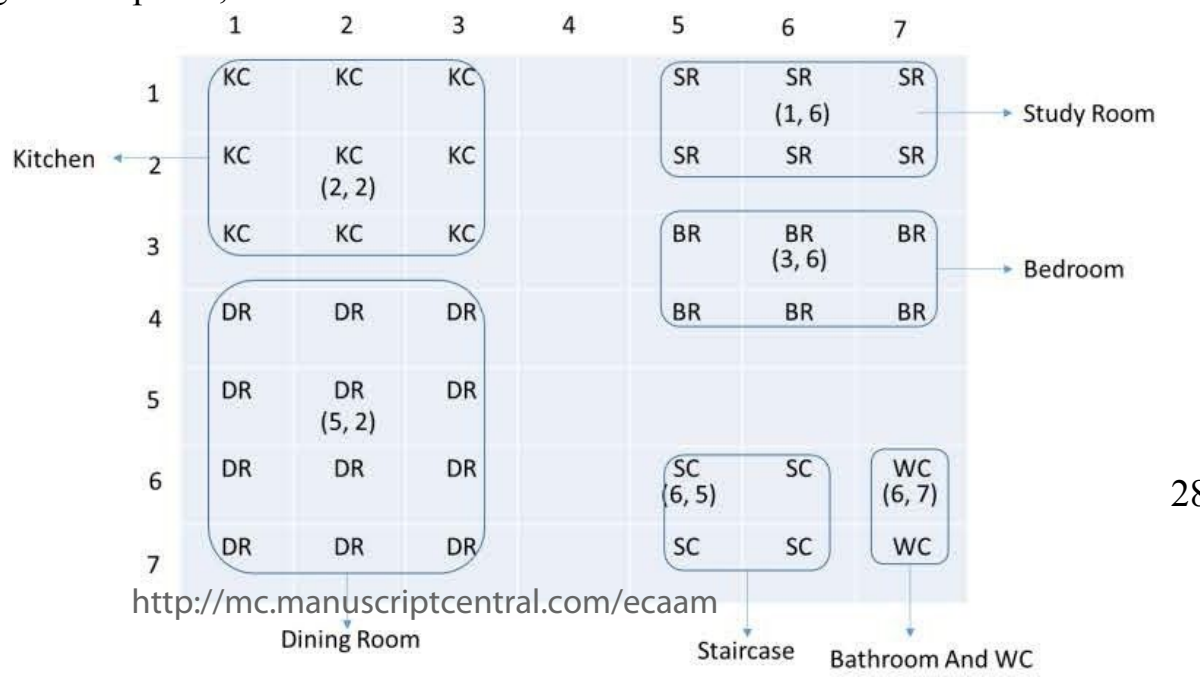


ii. After assigning the submatrices to the spaces, the following steps are conducted:

A. The total number of allocations for each square (building matrix entry) relates to the squares allocated to more than one room. Obviously, it is desirable for this number to be as small as possible, therefore, it is considered to be negative. This number is divided by the total building area for the purpose of normalisation;

B. The calculation of the Euclidean distance, if the criterion of the kitchen being in the centre of the building is activated. Obviously, it is desirable for this number to be as small as possible, therefore, the number is negative. For the purpose of normalisation, this number is divided by the greatest distance, i.e. the building's diameter;

C. The calculation of the Euclidean distance of the kitchen from the restroom, if the criterion of distancing the kitchen from restroom is activated. Obviously, it is desirable for this number to be as large as possible, therefore, the number is considered as a positive number. For the purpose of normalisation, this number is divided by the greatest distance, i.e. the building's diameter;

D. The calculation of the Euclidean distance of the centre of the stairs from the restroom, if the criterion of the closeness of the restroom to the stairs is activated. Obviously, it is desirable for this number to be as small as possible, therefore, the number is negative. For the purpose of normalisation, this number is divided by the greatest distance, i.e. the building's diameter;

E. The calculation of the Euclidean distance of the centre of the study room from the kitchen, if the criterion of distancing it from the kitchen is activated. Obviously, it is desirable for this number to be as large as possible, therefore, the number is considered as a positive number. For the purpose of normalisation, this number is divided by the greatest distance, i.e. the building's diameter;

F. Eventually all the above-mentioned values are added up to determine the related chromosome's value.

(f) Generating the new population through elitism, selection, mutation and crossover until a termination condition is reached:

i. Elitist selection guarantees that the solution quality acquired by the GA will not decrease from one generation to the next and is fixed at a certain percentage; 
ii. Selection will be carried out using tournament or roulette wheel selection;

iii. There is the possibility that in some generated alternatives, where some good submatrices are generated (spaces), but the overall fitness value is low and hence, performing crossover to fluctuate the programming of chromosomes could be highly beneficial. That is, a crossover between two partially good chromosomes could result in one with a high fitness value. One-point and two-point techniques are used for the crossover process;

iv. Mutation is used to sustain genetic diversity from one generation to the next and to allow for the algorithm to avoid 'local minima' by averting the chromosomes' population from becoming too similar to each other. Consequently, in the building matrix, some elements within the submatrices will be allocated to another submatrix.

2. The 'External Event' adopted in this prototype, which avoids DialogForm, allows users to interact with Revit and G-BIM at the same time. This will permit the user to check the sorted alternatives in 'TreeView' one by one as well as engage with the reuse/regeneration process. Moreover, the user can alter the parameters and view the resulted changes within the Revit interface;

3. The user can enter the mutation rate in TabPage as a GA parameter.

The following statements are made based on this stage of prototype development:

1. Two different methods of selection will be used in the next phase of prototype development and the user will be able to force one selection method to be performed manually;

2. Two different methods of crossover will be used in the next phase of prototype development and the user will be able to force one crossover method to be performed manually;

3. The user will be able to enter the elitism rate manually using G-BIM in the next prototype development phase;

4. The user will be able to alter a fixed number of generations for termination within the next phase of the prototype development;

5. The GA process can be saved at each stage and/or at different iterations; 
6. The fitness value can be viewed by the user for any alternative within any population

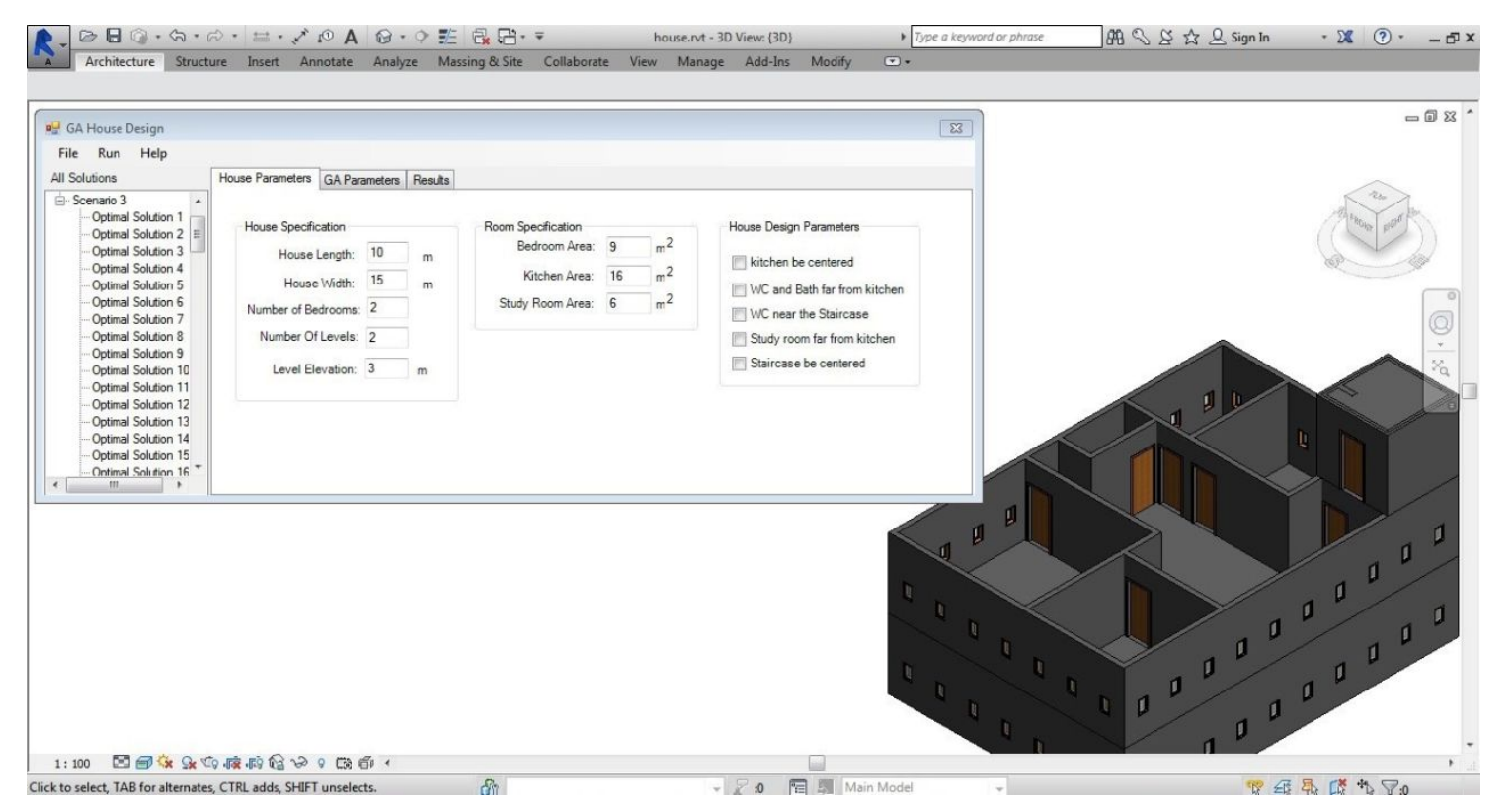

Figure 15: Input-form from the eighth phase of prototype development (with the House Parameters feature) 


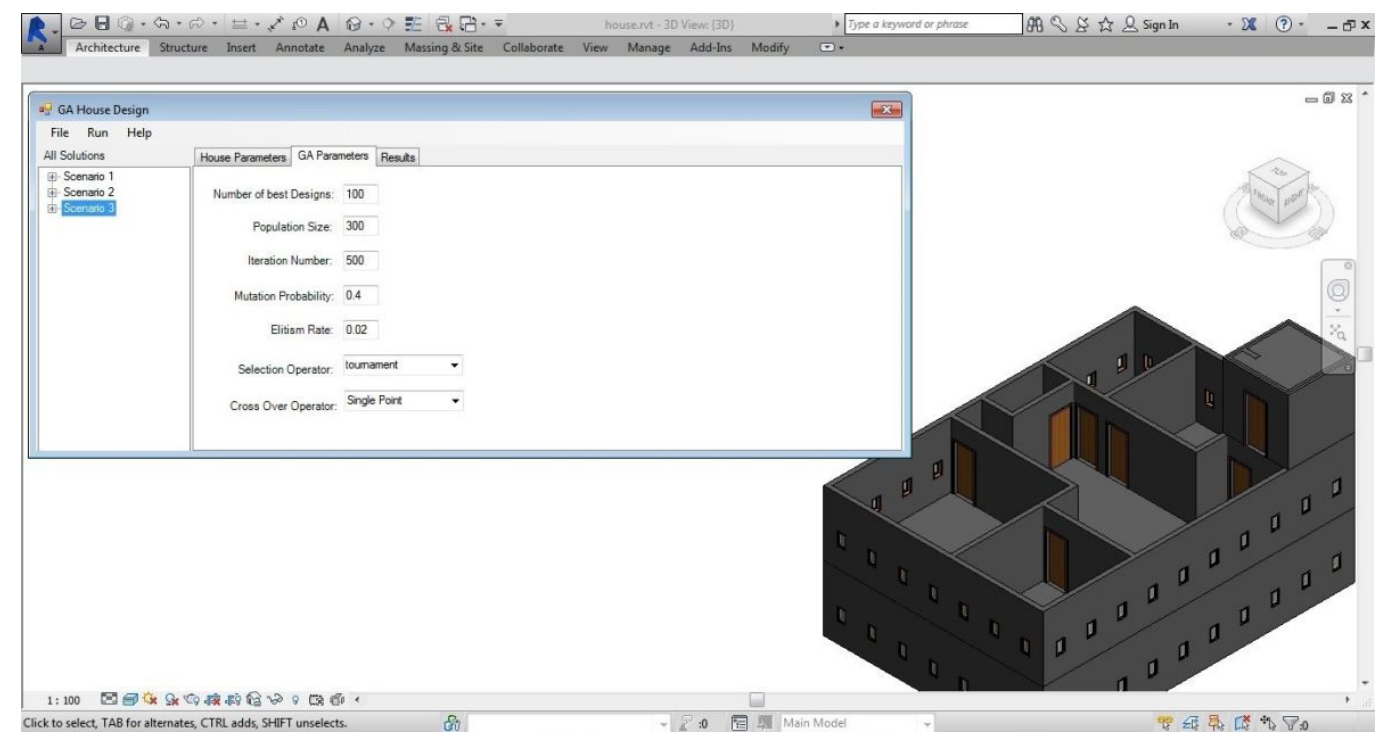

Figure 16: Input-form from the eighth phase of prototype development (with the GA Parameters feature)

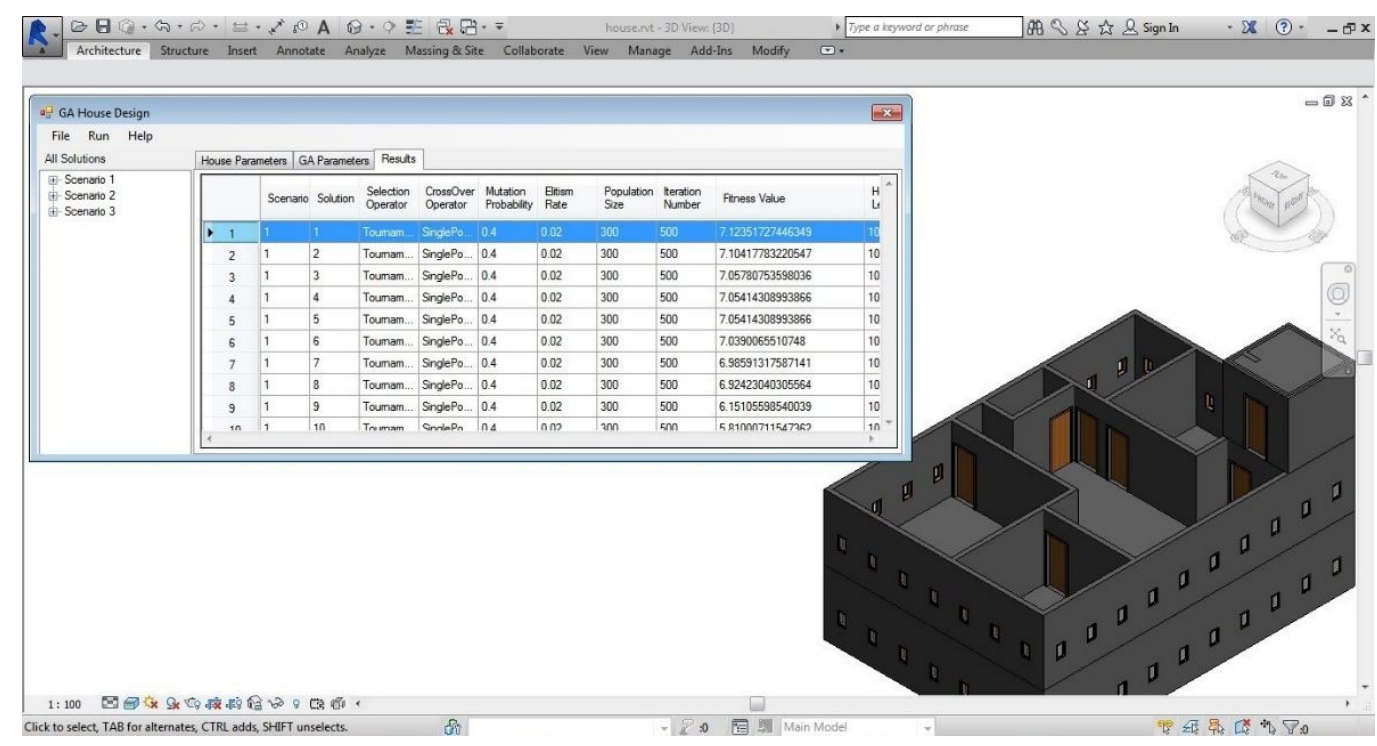

\section{Stage 8: User-interface}

For the eighth phase in the process of software prototyping, as presented in Figures 15, 16 and 17 , TreeView has been modified. As mentioned before, the results tab is added to the TabControl, which lets the user investigate alternatives at different stages of the GA process. The following statements are made based on this stage of the prototype development:

1. Different scenarios are shown as nodes in the TreeView panel. Generated (optimised) 
solutions are shown as a child chromosome under the specific node and the user can view each one within the Revit Node by clicking on it;

2. As it can be seen in Figure 16, the iteration number, elitism rate, selection operator (including tournament and roulette wheel) and cross-over operator (one-point and two-point) are added in the GA parameters' TabPage;

3. A GridView has been added to the Results TabPage (Figure 17). All the information (i.e. scenario number, solution number, selection operator, cross-over operator, mutation probability, elitism rate, population size, iteration number, fitness value, building size (length, width, and height)), each space size (length, width, and height) and the number of spaces, are stored in this GridView. Consequently, alternatives can be sorted by the user based on any of the aforementioned information.

\subsection{Prototype testing and validation}

Problem solving consists of programming and software development, with determination of the validity of the solution being part of any such activity. Moreover, applying development techniques and verification procedures throughout the development process leads to software quality achievement. In order to select a balanced collection of analysis, review and testing methods to adopt during the life cycle, careful reflection on specific quality elements and validation is required. Accordingly, identifying and resolving software issues early in the software lifecycle are primary goals.

\subsubsection{Focus group}

The main goal of the focus group was to investigate the participants' perception of G-BIM usage during the early stages of design. The analysis of the focus group discussions and debates allowed for the industry needs, concerns, and expectations concerning G-BIM adoption to be identified.

\section{Participants and the project}

In order to provide a better clarification of how G-BIM can be practically used in educational and professional settings, five individuals, including three postgraduate design students and two design practitioners with at least three years of experience, participated in a comparative focus group. The participants, whose fields of expertise were architecture, urban design, environmental design, and emergent 
technologies and design (Table 1), were asked to record the uses of the G-BIM prototype as they started on a given design project that included designing a three bedroom house for a young couple owning an estate located on St. Lawrence beach with total privacy. The participants were expected to design different parts of this house, including the living room, kitchen, bathroom (open style), two bedrooms, and a study room on two levels.

Table 1: Participants Details

\begin{tabular}{|c|c|c|c|c|}
\hline Name & Gender & Age & Educational Background & Position \\
\hline A1 & Male & 38 & Emergent Technologies (EmTech) & Architect \\
\hline A2 & Female & 45 & Environmental design & Designer \\
\hline S1 & Male & 28 & Architecture & PG Student \\
\hline S2 & Female & 26 & Architecture & PG Student \\
\hline S3 & Male & 30 & Architecture & PG Student \\
\hline
\end{tabular}

\section{Data collection}

The rationale of the data collection approach was to develop an instrument that was both reliable and user-friendly, thereby enabling the capturing the uses of G-BIM throughout the events rather than after them. Following this approach, participants were able to provide feedback. Hence, the research instrument designed in two parts, was the result of a self-evaluation report during the first session followed by group discussion once the second session had been completed. In this way, the selfevaluation report, that was sort of a diary, became a preliminary part for the group discussion (BURGESS and Coale, 1981). Thus, the two protocols, i.e. self-evaluation report and follow-up discussion (Schön, 1983), acted as complementary parts through which, the authenticity of the outcomes could be properly checked.

Self-evaluation report: During the first session, the self-evaluation report was arranged as a simple questionnaire containing a set of questions, each of which, denoted extracted design parameters that could be used later as G-BIM input. In addition, the researcher examined whether the self-evaluation report for G-BIM inputs could be reused. With this purpose in mind, a pilot study was carried out prior to the focus group. The questionnaire was constructed such that a G-BIM entry coding unit for six categories of conceptual design, namely, number of spaces, number of levels, number of zones, constraints, parameters, and relationships, was represented through each question in the questionnaire.

Follow-up discussion: Upon termination of the second session, the group discussion took place. The discussions targeted the G-BIM, and the way the participants had rated their designs from the first session as compared to the final one produced using G-BIM, their productivity during each session, and 
the G-BIM strengths and weaknesses. The follow-up discussions were conversations taking place between the participants and the researcher, rather than firmly pre-planned and structured questioning.

\section{System of the sessions}

The application of G-BIM was tested in two collaborative design sessions. Both sessions comprised a broad study of a design brief as well as the conceptual design automation context based on the given design brief (using G-BIM). Developing the application was not the main objective of these sessions, but rather, they were for validation and feedback.

In order for the participants to become acquainted with the design context, both sessions began with a design brief. They were then requested to analyse prior design briefs and production processes, subsequently being asked to draw out information from these analyses to keep in G-BIM by entering new links and features into the system's user interface, if they considered them essential and then, to continue with a design generation assignment. Designing, developing analytical and decision-making skills, internalising learning and tackling design problems were challenges that the participants faced using this technique. Participants were asked to obtain information and understanding regarding the design brief before attending each session. This was conducted in order to facilitate early generalisation of ideas, so that they could then apply them in similar situations in their design assignment (first session) or integrate them into G-BIM (second session). The identified information from the design brief was formalised, reshaped and reorganised into the manual design process. All the participants had experience and familiarity with the design context in both sessions but were also provided with assistance to help them in conceptualisation and generalisations.

Focus group first session: Throughout the first session, the participants were requested to develop their designs by getting familiarised with the design brief. In order to operationalise the design experiment, they could make use of both hand sketches and computational support for design development. A specific part of the assignment involved them sketching a memory map of their design decision process in addition to the design task, justifying for their problem formulations and providing the reason(s) behind their particular 
choice among the design alternatives that were developed earlier. In sum, the participants in the research were required to chronicle the steps in the design process leading to information and to further track the decisions made in the process of design development (Figure 18).

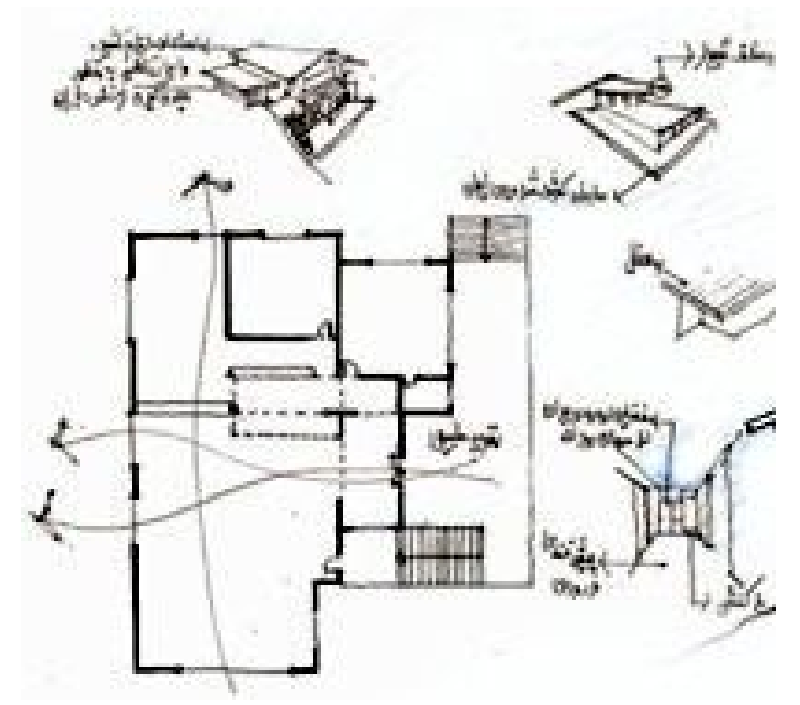

Figure 18: Sketches by A1

Focus Group Second Session: During the second session, the participants acquainted with the design context and the related terminology were asked to document a design brief based on G-BIM recognised input data. It was mandatory for them to elicit the information from the design brief and to amass it into the G-BIM database (based on their understanding) for the first cycle of design generation and use the generated designs from G-BIM for further alteration. Moreover, they were allowed to repeat the generation process on the basis of the findings or corrections of the initial cycle. It should be noted that they were required to make use of G-BIM from beginning to the end of the design process (Figure 19).

The assignment procedure of this study entailed every single participant simultaneously comparing the generated alternative(s) with those created during the first session. As the next step, both the formal and procedural conceptual design variables created by each participant were discussed. Finally, every single 
attendee was asked to discuss their design alternatives, among which a highly compatible one, able to satisfy all mutual restrictions, was chosen as a possible solution at each session. Moreover, for future accessibility, the eliminated design alternatives along with their partial solutions were stored in G-BIM. It should be noted that in this particular experiment, the participants demonstrated a higher degree of innovation and creativity in terms of both the design solution variety and the methods they formulated to attend to distinct restrictions and dependencies between tasks - evidenced by the following quotes:

"It was very simple and user-friendly tool and helped me come up with legible designs." (A2)

"[This tool] helped me explore the complexities of the project in generating more unique designs." (S3)

"This tool overlaid my perception of the possibility of automation at this stage [of design].” (A1)

“... satisfied most completely all attributes looking for: simple yet intelligent." (A2)

“... 'sci-fi' quality, hybrid between computational techniques and traditional ones." (S1)

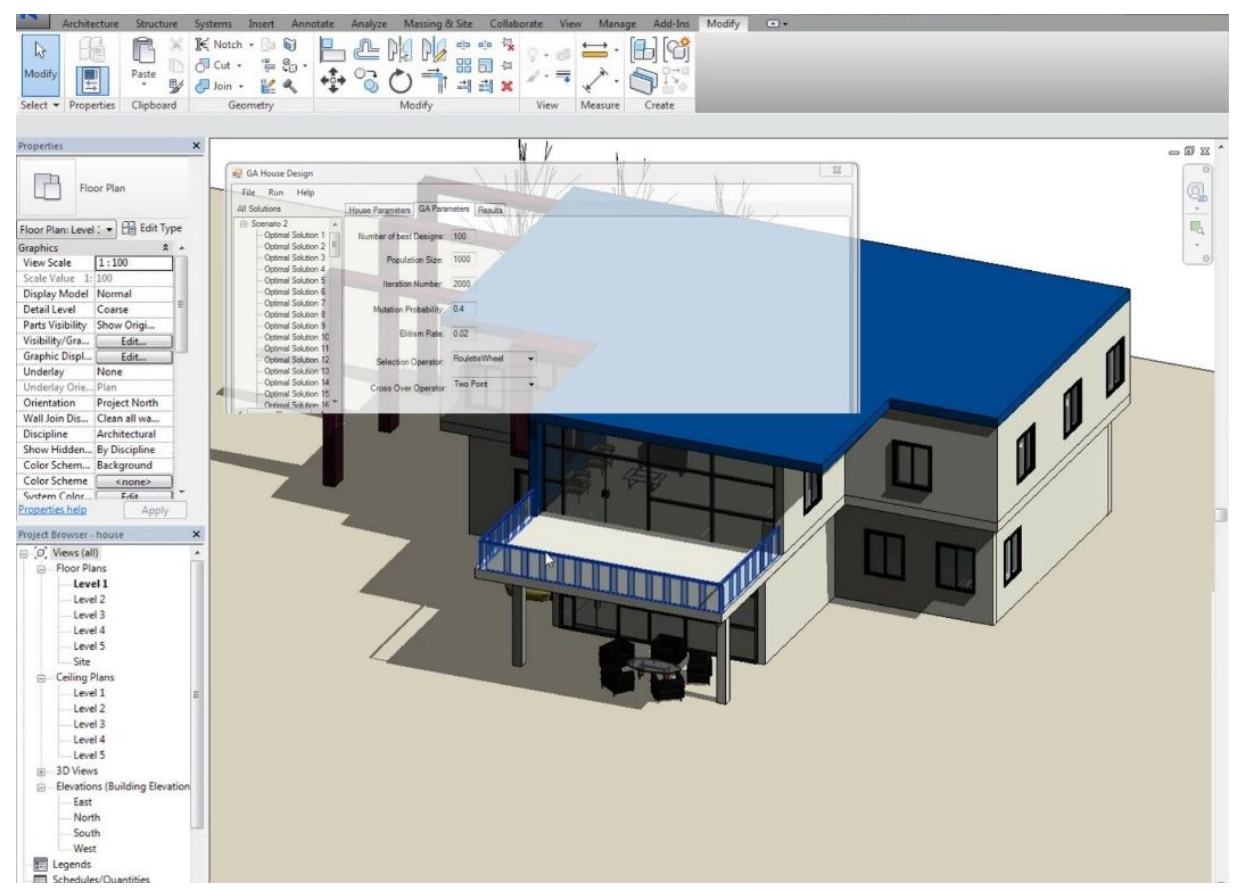

Figure 19: Final (modified) G-BIM model by A1 


\section{Comparing the Focus Groups Based on Participant Performances}

Participants' performance in the two sessions of the focus groups were assessed and compared in reference to their design output. The design alternatives were created by them in accordance with their experience, competence and perception of the optimisation during the first session. As a result, the information generated by them was relatively more solution specific, thus indicating that they faced greater difficulty in abstracting and generalising the information engendered. The second session, however, indicated a much-improved performance regarding abstract thinking and producing generalisable information content that could be stored and reused. Furthermore, in terms of creativity, it was observed that their orientation was more towards associating the concepts for the purpose of defining problems and creating varied strategies and concepts. Accordingly, in the second session, the participants demonstrated a higher degree of productivity concerning the development of alternative strategies, approaches and designs.

\section{Focus Groups Results}

Commercial BIM systems are product-oriented (accuracy and efficiency) rather than creativity-oriented (Abrishami et al., 2014a). In such systems, automating routine tasks, boosting drawing productivity, and cutting the cost of product development are top priorities. Considering BIM as a mere drawing tool (at design stage) is just a narrow definition of a technical drawing tool. However, G-BIM is a conceptual tool that can develop new ways of perceiving and conceiving design. In other words, using G-BIM may well encourage the development of new patterns, relationships, or aesthetics expanding without bringing about reduction in designers' creative options, as suggested by the participants when using computing for the purpose of conceptualising. G-BIM was repeatedly used for problem solving and formulation during the second phase of focus group, which elucidated the way a problem was formulated and ended in the final solution. In addition, what is currently the case is that the generation of various design types in G-BIM is not feasible. Therefore, enhancing the structuring of input data seems vital in problem formulation and solutions. 


\section{Conclusion}

The main proposition of this research concerned the inability of previous generative tools to embed information into generated alternative designs. Given the complexities of AEC projects, the industry is facing increasing pressure to become more integrated. In response to this, the adoption of automation has been increased by the industry. This includes offsite manufactured construction, with high product variety and significant variations in demand, which entails flexible and reconfigurable manufacturing systems, effective/cohesive supply chains as well as integrated and automatic modelling, simulation and decision support systems.

This paper explored the issues of process fragmentation and loss of design integrity. In doing so, it presented Generative BIM prototype as a potential solution for engaging with stakeholders at the early design phase of a project. This prototype provides techniques for exploring and generating design solutions, where models can be created with relevant links to all required information and details for the development process. The research methodological approach followed the CIFE "horseshoe" methodology and engaged a focus group of five AEC professionals. Research findings identified that G-BIM was able to capture project design information and creating optimal design solutions. However, it was acknowledged from the outset that there was a need to address the entire requirements for BIM Level 3 into a fully integrated system. This in itself poses additional challenges from an end-user 
perspective, as predominantly, end-users are not typically programmers, nor experts in writing genetic algorithms.

The proposed G-BIM prototype and scientific findings offer new insight and understanding into the integration of evolutionary design (to optimise design solutions at the conceptual design stage). Specific findings include: i) the provision of specific techniques used for exploring and generating design solutions; ii) methods of designing and incorporating generative processes with design complexity and high levels of variability; iii) an approach through which stakeholders can more meaningfully engage in collaborative environments through conceptual design phases - enabling both short-term asynchrony and long-term asynchrony. However, from a research limitation perspective, it is equally acknowledged that this prototype is continually being interactively refined to improve internal and external consistency and reliability, cognisant of homogeneity, generalisability and repeatability.

Due to its expansion beyond the time limits, the final prototype has not yet been fully examined in a range of real-life design settings. In order to achieve a successful and adequate implementation of the prototype, its capacity to adjust to variable contexts and different AEC disciplines is essential. Improving G-BIM is aimed at in future, through user participation and their reflection on the design process along with propositions for its further enhancement in each context of use. The successful progress of similar systems in design has become possible via these generalised viewpoints and propositions.

\section{References:}

Abanda, F. H., Tah, J. H. M., \& Cheung, F. K. T. (2017). BIM in off-site manufacturing for buildings. Journal of Building Engineering, 14(Supplement C), 89-102. doi:https://doi.org/10.1016/i.jobe.2017.10.002

Abrishami, S., Goulding, J., Pour Rahimian, F., \& Ganah, A. (2015). Virtual Generative BIM Workspace for Maximising AEC Conceptual Design Innovation: A Paradigm of Future Opportunities. Construction Innovation, 15(1), null-null. 
Abrishami, S., Goulding, J., Rahimian, F. P., \& Ganah, A. (2014a). Integration of BIM and generative design to exploit AEC conceptual design innovation. Information Technology in Construction, 19, 350-359.

Abrishami, S., Goulding, J., Rahimian, F. P., Ganah, A., \& Sawhney, A. (2014b). G-BIM Framework and Development Process for Integrated AEC Design Automation. Procedia Engineering, 85(0), 10-17.

Arayici, Y., Fernando, T., Munoz, V., \& Bassanino, M. (2018). Interoperability specification development for integrated BIM use in performance based design. Automation in Construction, 85(Supplement C), 167-181. doi:https://doi.org/10.1016/j.autcon.2017.10.018

BURGESS, A., \& Coale, S. (1981). An Interview with Anthony Burgess. Modern Fiction Studies, 27(3), 429-452.

Cha, H. S., \& Lee, D. G. (2015). A case study of time/cost analysis for aged-housing renovation using a pre-made BIM database structure. KSCE Journal of Civil Engineering, 19(4), 841-852. doi:10.1007/s12205-013-0617-1

Damen, T., Sebastian, R., MacDonald, M., Soetanto, D., Hartmann, T., Di Giulio, R., Bonsma, P., \& Luig, K. (2015). The Application of BIM as Collaborative Design Technology for Collective SelfOrganised Housing. International Journal of 3-D Information Modeling (IJ3DIM), 4(1), 1-18. doi:10.4018/IJ3DIM.2015010101

Elghaish, F., \& Abrishami, S. (2020). Developing a framework to revolutionise the 4D BIM process: IPD-based solution. Construction Innovation.

Elghaish, F., Abrishami, S., \& Hosseini, M. R. (2020a). Integrated project delivery with blockchain: An automated financial system. Automation in Construction, 114, 103182. doi:https://doi.org/10.1016/j.autcon.2020.103182

Elghaish, F. A. K., Abrishami, S., Hosseini, M. R., \& Abu-Samra, S. (2020b). Revolutionising cost structure for integrated project delivery: a BIM-based solution. Engineering, Construction and Architectural Management.

Ercan, B., \& Elias-Ozkan, S. T. (2015). Performance-based parametric design explorations: A method for generating appropriate building components. Design Studies, 38(Supplement C), 33-53. doi:https://doi.org/10.1016/j.destud.2015.01.001

Frazer, J. (2016). Parametric computation: History and future. Architectural Design, 86(2), 18-23.

Frazer, J. H. (2002). Creative Evolutionary Systems. In P. Bentley \& D. Corne (Eds.), (pp. 253-274253-274): Morgan Kaufmann.

Fruchter, R., Herzog, S., Hallermann, N., \& Morgenthal, G. (2016). Drone Site Data for Better Decisions in AEC Global Teamwork. Paper presented at the 16th International Conference on Computing in Civil and Building Engineering, Osaka.

Goulding, J. S., \& Rahimian, F. P. (2012). Industry Preparedness: Advanced Learning Paradigms for Exploitation. In Construction Innovation and Process Improvement (pp. 409-433).

Jacobson, I., Booch, G., \& Rumbaugh, J. (1999). The Unified Software Development Process: ADDISON-WESLEY Longman, Inc. Reading.

Janssen, P. (2006). A Generative Evolutionary Design Method. Digital Creativity, 17(1), 49-63-49-63.

Morgan, D. L. (1997). Focus groups as qualitative research: Sage.

Narahara, T. (2007). Enactment software: spatial designs using agent-based models. Complex Interaction and Social Emergence. 
Narahara, T., \& Terzidis, K. (2006). Multiple-constraint genetic algorithm in housing design.

Parker, A., \& Tritter, J. (2006). Focus group method and methodology: current practice and recent debate. International Journal of Research and Method in Education, 29(1), 23-37.

Pilechiha, P., Mahdavinejad, M., Pour Rahimian, F., Carnemolla, P., \& Seyedzadeh, S. (2020). Multiobjective optimisation framework for designing office windows: quality of view, daylight and energy efficiency. Applied Energy, 261, 114356. doi:https://doi.org/10.1016/j.apenergy.2019.114356

Pour Rahimian, F., Chavdarova, V., Oliver, S., \& Chamo, F. (2019). OpenBIM-Tango integrated virtual showroom for offsite manufactured production of self-build housing. Automation in Construction, 102, 1-16. doi:https://doi.org/10.1016/i.autcon.2019.02.009

Pour Rahimian, F., Ibrahim, R., \& Baharudin, M. N. (2008). Using IT/ICT as a new medium toward implementation of interactive architectural communication cultures. Paper presented the Proceedings - International Symposium on Information Technology 2008, ITSim.

Pour Rahimian, F., Seyedzadeh, S., Oliver, S., Rodriguez, S., \& Dawood, N. (2020). On-demand monitoring of construction projects through a game-like hybrid application of BIM and machine learning. Automation in Construction, 110, 103012. doi:https://doi.org/10.1016/j.autcon.2019.103012

Rhodes, C. (2019). Construction industry: statistics and policy. Retrieved from House of Commons Library:

Schön, D. A. (1983). The Reflective Practitioner: How Professionals Think in Action: Basic Books.

Seyedzadeh, S., Pour Rahimian, F., Rastogi, P., \& Glesk, I. (2019). Tuning machine learning models for prediction of building energy loads. Sustainable Cities and Society, 47, 101484. doi:https://doi.org/10.1016/j.scs.2019.101484

Seyedzadeh, S., Rahimian, F., Oliver, S., Glesk, I., \& Kumar, B. (2020). Data Driven Model Improved by Multi-Objective Optimisation for Prediction of Building Energy Loads. Automation in Construction, In Press.

Skibniewski, M. J. (2014). Information technology applications in construction safety assurance. Journal of Civil Engineering and Management, 20(6), 778-794.

Succar, B., \& Poirier, E. (2020). Lifecycle information transformation and exchange for delivering and managing digital and physical assets. Automation in Construction, 112, 103090.

Walasek, D., \& Barszcz, A. (2017). Analysis of the Adoption Rate of Building Information Modeling [BIM] and its Return on Investment [ROI]. Procedia Engineering, 172(Supplement C), 12271234. doi:https://doi.org/10.1016/i.proeng.2017.02.144

Wasserman, A. I., Pircher, P. A., Shewmake, D. T., \& Kersten, M. L. (1986). Developing interactive information systems with the User Software Engineering methodology. IEEE Transactions on Software Engineering, SE-12(2), 326-345-326-345. 